\title{
Smart invasome synthesis, characterizations, pharmaceutical applications, and pharmacokinetic perspective: a review
}

\author{
Sopan Nangare ${ }^{1 *}$ (D) and Shailesh Dugam ${ }^{2}$
}

\begin{abstract}
Background: Scientists are constantly looking for the introduction of unique drug delivery systems for the existing drug molecule. Since the skin is one of the primary and essential organs of the human body, it needs successful research development for the delivery of the drug. While the skin is assumed a human body's multifunctional organ, it has minimal permeability across the stratum corneum (SC). Since this is an influential barrier for the active agent, several carrier platforms to surmount this obstacle have been created. Invasomes are the liposomal vesicles, which incorporate small quantities of ethanol and terpenes or a mixture of terpenes, as potentials for improved penetration of the skin. The rate of penetration of invasomes through the skin is significantly greater than that of liposomes and ethosomes. Invasomes focus on providing a series of benefits namely enhanced drug effectiveness, increased conformity, and ease for patients.
\end{abstract}

Main body: The present article portrays insights of invasomes which include composition and preparation methods of invasomes. The article gives a brief review of the penetration mechanism, synthesis process, and characterizations of invasomes. The article gives a point by point audit about pharmaceutical applications, viz. anticancer, antihypertensive, anti-acne, vitamin analog, anticholinergic, antioxidant, etc. The pharmacokinetic properties of invasomes have also been described.

Conclusion: The key goal of an invasome-based delivery system is not only to strengthen the efficacy and safety of the drug but also to dramatically increase patient conformity and the therapeutic value to a significant extent. The delivery of drugs via the skin membrane in advanced drug delivery systems is a fascinating fact. Many pharmaceutical studies have shown that plentiful drug molecules are less soluble, have less bioavailability and stability, have less penetration, etc. Therefore, a new form of dosage with exceptional characteristics like invasomes can be created.

Keywords: Invasome, Penetration enhancer, Pharmaceutical applications, Terpene, Drug delivery

\section{Background}

The transdermal route is an effective way to localized or systemic effects. The drug delivery systems based on the nanocarrier have gained optimistic renovation, in particular for the drug delivery through the skin as a non-

\footnotetext{
* Correspondence: snangareopan@gmail.com

1 Department of Pharmaceutical Chemistry, H. R. Patel Institute of

Pharmaceutical Education and Research, Shirpur, Dhule, MS 425405, India

Full list of author information is available at the end of the article
}

invasive route. It improves essential drug/bioactive candidate penetrant properties. To date, distinct nanocarriers (nanovesicles) have been adopted for enhancing the skin membrane permeation rate of drug candidates [1]. Drug delivery through the skin membrane provides substantial improvements over traditional methods including invasive and oral routes. It helps to bypass the first-pass metabolism and increases patient compliance [2]. Furthermore, it shows fewer systemic side effects and offers targeted 
delivery of drugs along with painless administration of dosage form [3]. Unfortunately, the low diffusion rate of drugs across $\mathrm{SC}$ is a major barrier for topical/transdermal drug delivery. Literature mentioned that $\mathrm{SC}$ is a part of the skin that serves as a barrier for drug diffusion from the site of administration to the systemic circulation [4]. In brief, the $\mathrm{SC}$ is an outer layer of skin having keratinrich corneocytes and lipid lamellae [5]. This thorny crystalline lipid lamella offers a crucial barrier characteristic to the skin membrane [6]. Therefore, tremendous efforts have been taken to weaken and disrupt the SC using mechanical and chemical methods viz. iontophoresis and employment of penetration enhancers, respectively $[7,8]$. For the last couple of decades, the delivery of drug/biomolecule through skin membrane has been archived using various nanovesicles viz. liposome, nanoparticles, micelle, micro-emulsion, dendrimers, etc. [9]. In recent years, penetration enhancers containing novel elastic phospholipid vesicles including transfersomes $[9,10]$ ethosomes [9], and invasomes [9] have been extensively adopted for the delivery of actives/drug through the skin [1].

\section{Main text}

\section{Invasomes}

The typical liposomes (phospholipid vesicles) contain lipids (cationic, anionic, and neutral) and cholesterol that increase the encapsulation of pharmaceutical (hydrophilic, lipophilic, amphiphilic) active components. In the case of liposomes, the hydrophilic drug is placed inside the watery heart, lipophilic inside the lipid bilayer, and amphiphiles are placed in the interlayer of liposomes [11]. Unfortunately, suitable carrier liposome has little (or no) value, as liposomes do not penetrate the skin intensely. It is also limited by SC and ineffective for the skin to deliver drugs [12]. Therefore, liposomes are suffering from the major restriction that creates incertitudes about the global application of liposomes [13, 14]. Overall, there is a necessity to develop advanced carriers for efficient delivery of active pharmaceutical ingredients through the skin (dermis, epidermis, subcutaneous, etc.). In recent times, the terpenes (single or ratio of terpenes), ethanol, etc. have been incorporated in a lipidic vesicular system (viz. invasomes) which enhanced the considerable penetration of vesicles (with active molecules) through the skin [14]. Fascinatingly, invasomes are novel liposome-associated vesicles (Fig. 1). Invasomas are bilayer vesicles, comprised of soya phosphatidylcholine (SPC), lysophosphatidylcholine (flexibility substances), terpenes, and ethanol (permeation enhancer). The composition of the invasome is depicted in Fig. 2. Synergistic changes in all these components (i.e., unsaturated phospholipids, terpenes, and ethanol) are accomplished in deeper skin drug levels than in the case of traditional liposomes or a drug solution. Verma et al. (2004) acknowledged this when researching invasomal cyclosporine-A deposition and achieving promising results in alopecia areata therapy $[9,15,16]$. Particularly, this is a subset of liposomes. Because of penetrative boosters (terpene and ethanol), invasomes offer high penetration potential [17]. According to the literature, invasomes consist of phosphatidylcholine, ethanol, and terpene/combination of terpenes [18-20] and all these parts possess a significant function in invasomes like bilayer forming (phosphatidylcholine) and edge activation (lysophosphatidylcholine), and penetration enhancement (ethanol, terpene). Particularly, it is important to note that bilayer components give vesicles "rigidity" or "fluidity." Surveys stated that the rise in invasive vesicle

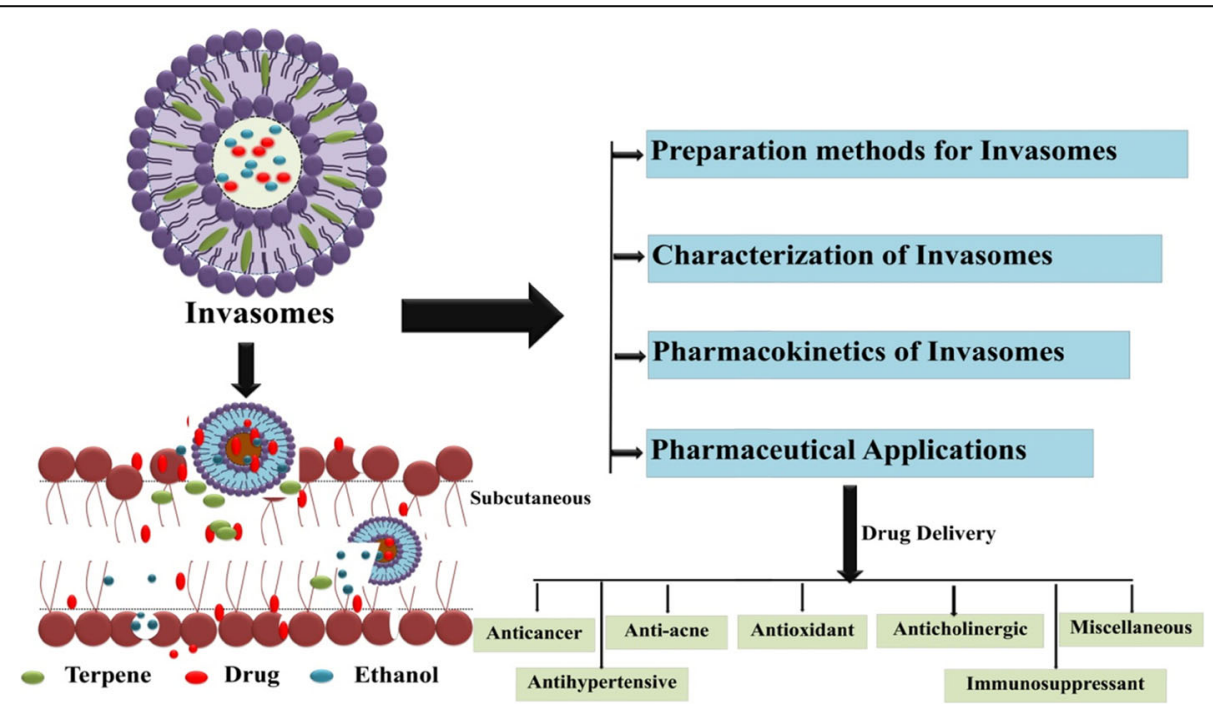

Fig. 1 Pharmaceutical applications of invasomes 


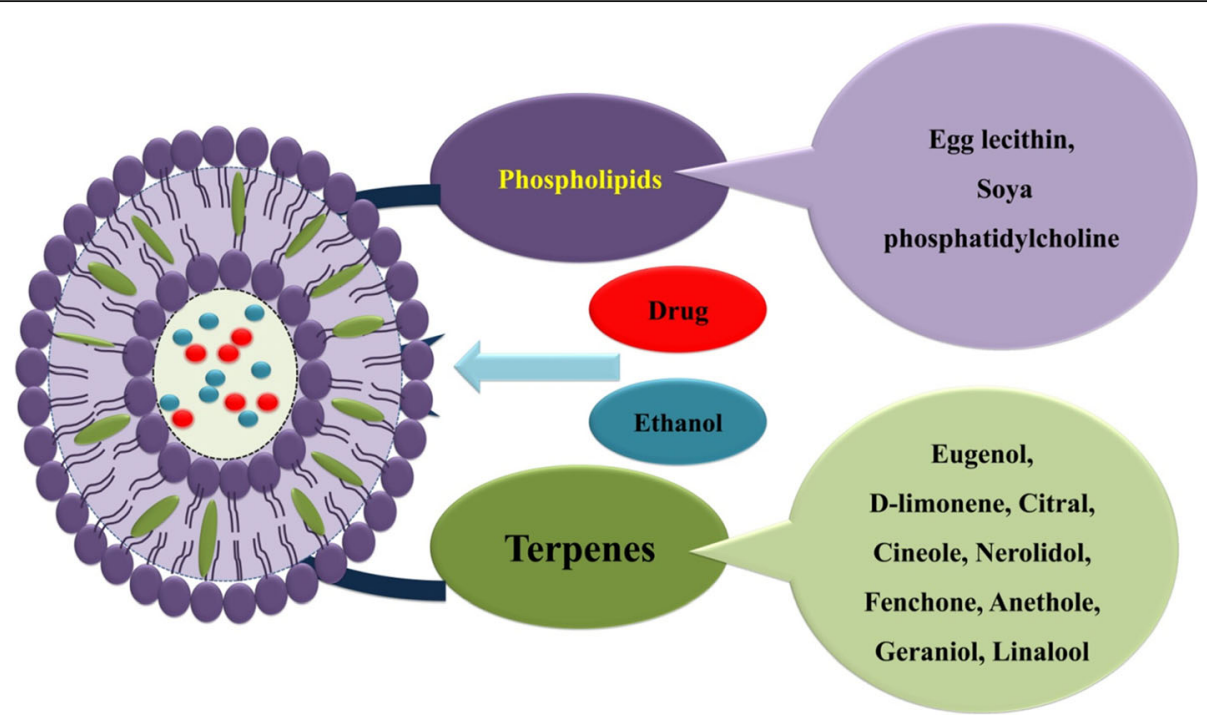

Fig. 2 Composition of invasomes

penetration is directly dependent on the fluidity and elasticity of the bilayer of lipid vesicles. Plenty of publications stated that ultra-flexible lipid vesicles which are much smaller than vesicles can respond to an applied stimulus by quickly deforming the shape and passing through skin pores. For maximum deformation of vesicle membrane, specific types and concentrations of edge activators are needed $[21,22]$. The incorporation of ethanol and terpene in invasomes facilitates lipid fluidity in the vesicle structure and produces a flexible structure which is less rigid than typical liposomes [23, 24]. The incorporation of a bilayer-forming agent, edge activator, and penetration enhancers give the synergistic effect and offers the superior penetration power to the drug/active, improves the flexibility and fluidity of invasomes [16, 25]. Briefly, the soya-phosphatidylcholine can create an invasomal bilayer matrix, whereas, the lysophosphatidyl choline acts as an edge activator, which provides flexibility to the phosphatidylcholine bilayers $[9,26]$. On the other hand, the addition of ethanol and terpenes act as penetration enhancers for drugs. Moreover, it endows fluidity/ flexibility with the phospholipid bilayers. In the case of ethanol, ethanol contact with lipids in the SC polar group region induces structural changes in keratinized / lipophilic regions, decreases in the lipid transition temperatures, and thus the tightly packaged SC lipids are fluidized and disturbed. Furthermore, ethanol presence facilitates the flexibility of the intercellary lipid matrix because lipid acyl chains have rotating freedom. Accordingly, it increasing the fluidity of lipids and this results in the shape of the vesicle that has more soft and less rigid than traditional liposomes. In addition to improved penetration, ethanol generates distinctly negative surface load and minimal vesicular aggregation due to electrostatic repulsion, which contributes to increased invasive stability under conditions of storage [9]. Other penetration enhancers including terpenes induce greater penetration of the drug by breaking down near bilayers and lipid packing in the SC. In addition, breaking down the hydrogen bonds and separating SC lipids, rising the partition into SC through boosting lipid fluidity and rising diffusion through intercellular lipids are also reports of increasing active permeability with terpens $[15$, $16,25,27]$. In case of low ethanolic content in invasomes, because of the presence of terpenes in invasome, it alter the SC properties. Accordingly, it helps to deliver the drug into deeper regions of the skin [27-30]. Owing to such versatile properties of invasomes, it offers the way to deliver hydrophilic and hydrophobic drug/ biomolecule delivery across the dermal and transdermal region. Despite of this, various advantages and disadvantages associated with the invasomes [31] are reported in Table 1. Despite the remarkable advantages of invasomes, there is some major concern regarding concentration and types of penetration enhancers. The concentration of terpene have been observed as vary in with respective formulation (Table 2). Additionally, mostly naturaly obtained terpene are the most effective and safe classess of penetration enhancers. While penetration enhancements work properly in transdermal delivery, few are clinically authorized for their skin irritation and toxicity. Therefore, it is worth remembering that the balancing of the potency and toxicity of terpene or terpene mixtures for human is an important regulatory requirement during the development of invasome formulations $[15,16,25,27]$. 
Table 1 Invasome advantages and disadvantages

\begin{tabular}{ll}
\hline Advantages & Disadvantages \\
\hline $\begin{array}{l}\text { 1. It is a non-invasive drug delivery technique. } \\
\text { 2. It enhances the penetration rate of actives. }\end{array}$ & $\begin{array}{l}\text { 1. It requires a high cost for production } \\
\text { 2. Chance of leakage and fusion of encapsulated active. } \\
\text { 4. More patient compliance (cream/gel). }\end{array}$ \\
$\begin{array}{l}\text { 5. Invasome contains non-toxic excipients. } \\
\text { 3. Invasome containing phospholipids may get oxidized/hydrolyzed } \\
\text { and affect the stability of vesicles. }\end{array}$ \\
\hline
\end{tabular}

\section{Preparation methods for invasomes}

As per the literature, few methods are described for the proposed novel invasome drug carriers. The most famous approach is the technique of mechanical dispersion and film hydration.

\section{Mechanical dispersion technique}

In the case of the mechanical dispersion technique, the active drugs/biomolecule and terpene or a mixture of terpenes are dissolved in phospholipid containing ethanol. After proper mixing, the mixture should vortexed (5 min) and sonicated (5 min) to provide a simple solution. After that, the phosphate buffer ( $\mathrm{pH}$ 7.4)/phosphate buffer saline (PBS)/suitable solvent added to the solution for hydration of vesicles by applying a syringe with constant vortexing $(5 \mathrm{~min})$. Finally, the solution was sifted for extrusion of multilamellar vesicles via polycarbonate membranes of pore size ranges $(400 \mathrm{~nm}, 200 \mathrm{~nm}, 100$ $\mathrm{nm}, 50 \mathrm{~nm}$ ) and repeated several times [32, 44]. The preparation of invasome by employing mechanical dispersion is depicted in Fig.3.

\section{Film hydration technique}

In the film hydration technique, the mixture of ethanol and phospholipid dissolved in a mixture of methanol and chloroform $(2: 1 \mathrm{v} / \mathrm{v})$. This mixture dried by adopting a rotary flash evaporator by lowering the pressure $(500$ to $1 \mathrm{mbar}$ ) for $2 \mathrm{~h}\left(\right.$ at $50^{\circ} \mathrm{C}$ ). After that, the film was kept for $2 \mathrm{~h}$ under 1 -mbar pressure and accompanied by a nitrogen flush. The PBS (pH 7.4) or mixture with terpenes, ethanol, and PBS can be selected for the hydration of deposited film for $30 \mathrm{~min}$. Finally, after cooling the mixture, the terpene/ mixture of terpenes and ethanol should be added to obtain the invasome vesicles. The prepared invasome was vortexed followed by ultrasonication and seized by using extrusion via polycarbonate membranes of the various pore size range several times $[15,45]$. The formation of invasome by applying the film hydration method is represented in Fig.4.

\section{Characterizations of Invasomes Vesicle shape}

The invasome vesicle size can be determined by using various advanced microscopic techniques such as scanning electron microscopy (SEM) and transmission electron microscopy (TEM). An ample literature survey claimed that the invasomes could be set up to formulate into an unfamiliar shape. The TEM images of isradipineloaded invasome vesicles were reported comprising of spherical shape, smooth, and even surface morphology [26]. Cryo-TEM images of vesicular systems of idebenone- and azelaic acid-loaded invasomes were reported to be unilamellar vesicles showing a mixture of small unilamellar vesicles (SUVs) and large unilamellar vesicle (LUVs) along with a few oligolamellar vesicles [25]. Other Cryo-TEM images (Fig. 5) of temoprofin invasome vesicles were reported to be uni- and bi-lamellar [33]. They reported the SEM images of finasterideloaded invasomes were found to be spherical and unilamellar [30]. To sum up, the drug-loaded invasome vesicle is found as an in deformable, spherical shape along with individual or mixture of uni-, bi-, and oligolamellarity.

\section{Vesicle size and polydispersity index}

Drug-loaded invasome vesicle size has been determined using dynamic light scattering (DLS) and photon correlation spectroscopy. The minoxidil-loaded invasomes showed a size range between $140 \mathrm{~nm}$ to $195 \mathrm{~nm}$. Besides this, addressed the size of invasome vesicles to enhance with vesicle surface potential of hydrophilic molecules (example: labrasol, and transcutol) [35]. In the case of isradipine-loaded invasome vesicle size, the invasomes with $0.1 \%$ b-citronellene shows a small particle size as compared with the $0.5 \%$ terpene [26]. Finasteride-loaded invasomes reported that the vesicle size of invasome can fluctuate with terpene types. The carvone, nerolidol, limonene-based invasome were found in the size of $4.54 \mu \mathrm{m}, 11.23 \mu \mathrm{m}, 4.8 \mu \mathrm{m}$, respectively, which is merely based on the molecular weight of terpene [30], whereas the temoprofin-loaded invasomes were informed that the standard mixture of terpene also efficiently affects the vesicle size [15]. Hence, invasome vesicle size can change based on individual terpene, terpene mixture, and based on its molecular weight. The polydispersity index (PDI) of invasomes was reported to be $<0.3$, which is supported by the homogeneous and monodisperse suspension of invasomes [35]. Besides, the PDI of the investigated temoprofin invasomes was in the range from $0.066(1 \% \mathrm{w} / \mathrm{v}$ cineol $)$ to $0.144 \quad(1 \%, \mathrm{w} / \mathrm{v}$ terpene 


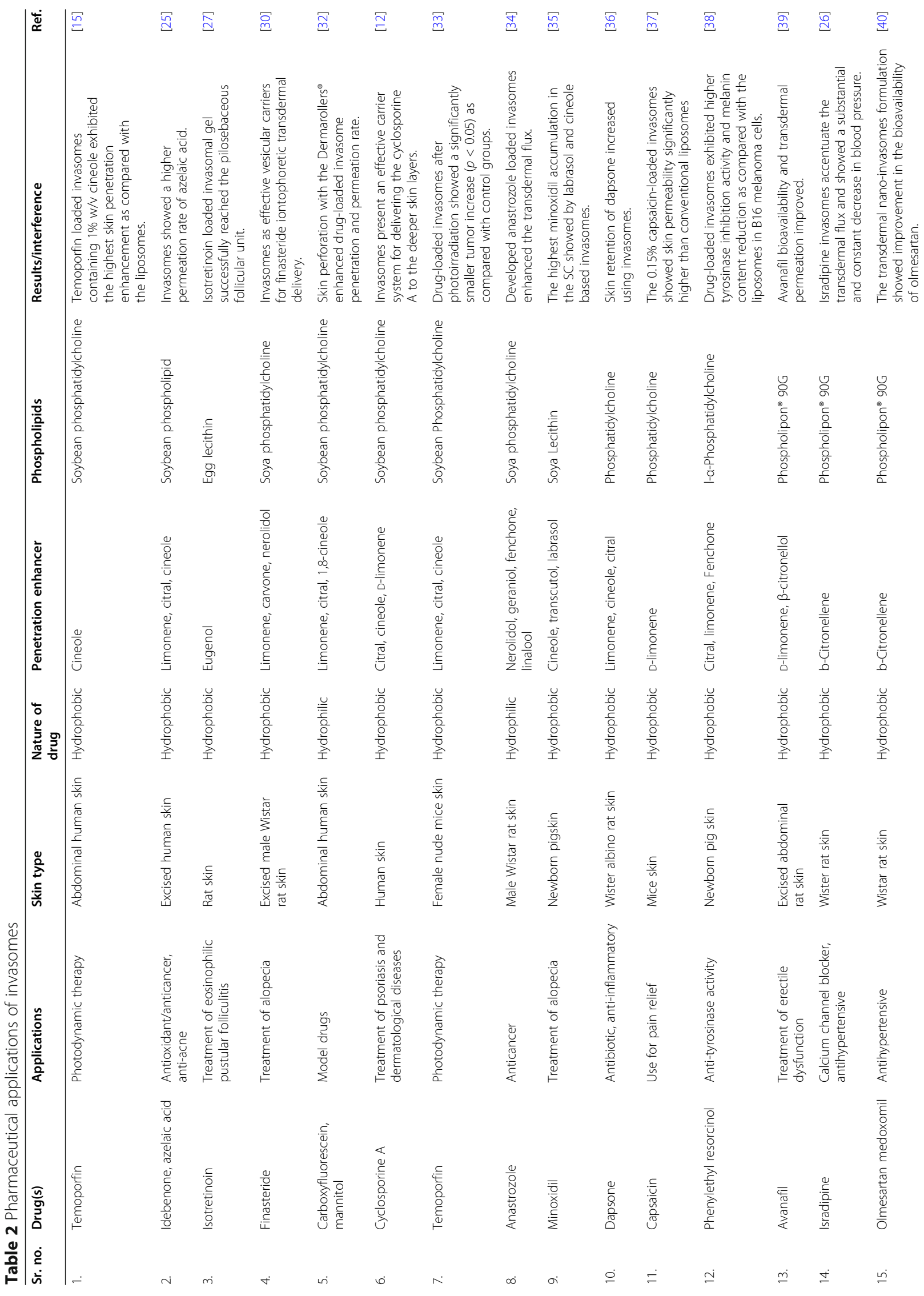




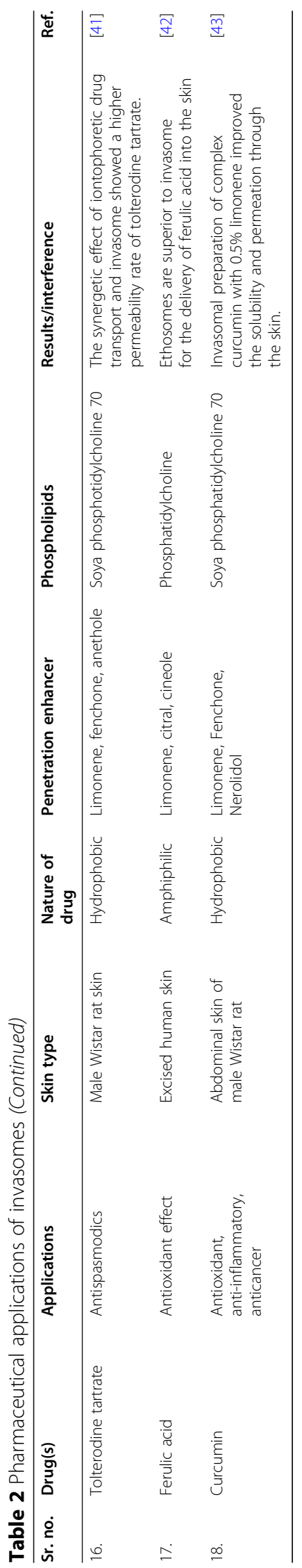




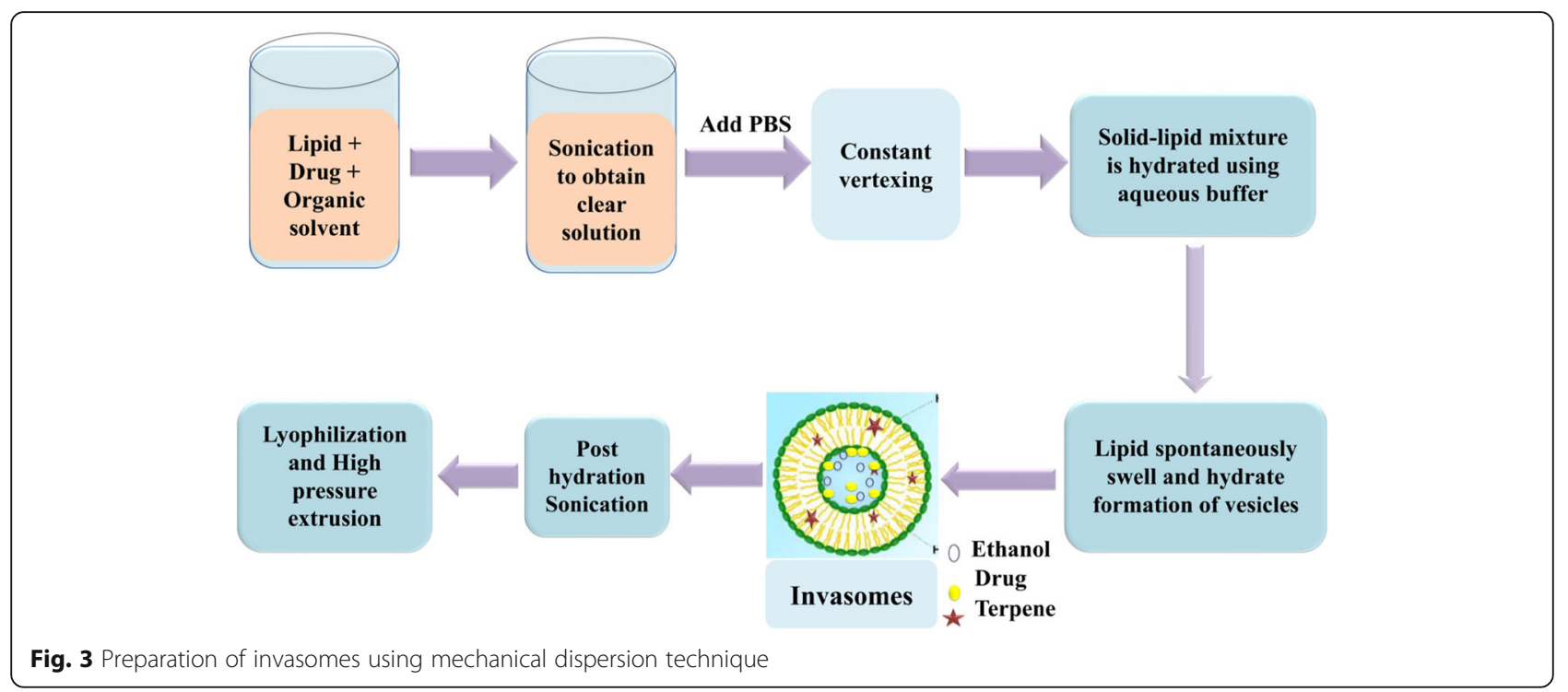

mixture), that concluded the homogeneous populations of vesicles [15]. Overall, the PDI of invasomes is more important to obtain a monodisperse, homogeneous distribution of invasomes in the formulation.

\section{Zeta potential}

Zeta potential of drug-loaded invasomes can be calculated using Zetasizer. The literature claimed that the negative potential of vesicles can enhance the penetration rate of the drug [2]. Additionally, the admirable zeta potential indicates the stability of invasomal formulation. For example, Vidya et al. have been developed the anastrozole-loaded invasomes, which exhibited a negative zeta potential $(-20.9 \mathrm{mV})$. Interestingly, the negative potential of the invasome vesicles may because of the presence of ethanol. Further, it rules out the vesicle aggregation because of its electrostatic repulsive forces that resulted in the invasomal dispersion stability [34]. In 2013, Prasanthi et al. have been reported that the increased (0.5 to $1.5 \%)$ nerolidol percentage resulted in the improvement in zeta potential (-67.5 to $-71.4 \mathrm{mV})$. Whereas when the carvone concentration increased from 0.5 to $1.5 \%$, the zeta potential decreased from -73.3 to $-63.3 \mathrm{mV}$. On this account, they have mentioned that the zeta potential changes with terpene type and percentage incorporated were not more than $\pm 5 \%$ [30]. Therefore, to avoid the vesicle aggregation and good invasome stability in dispersion/suspension like formulations, the zeta potential of invasomes is more important and it should be optimum.

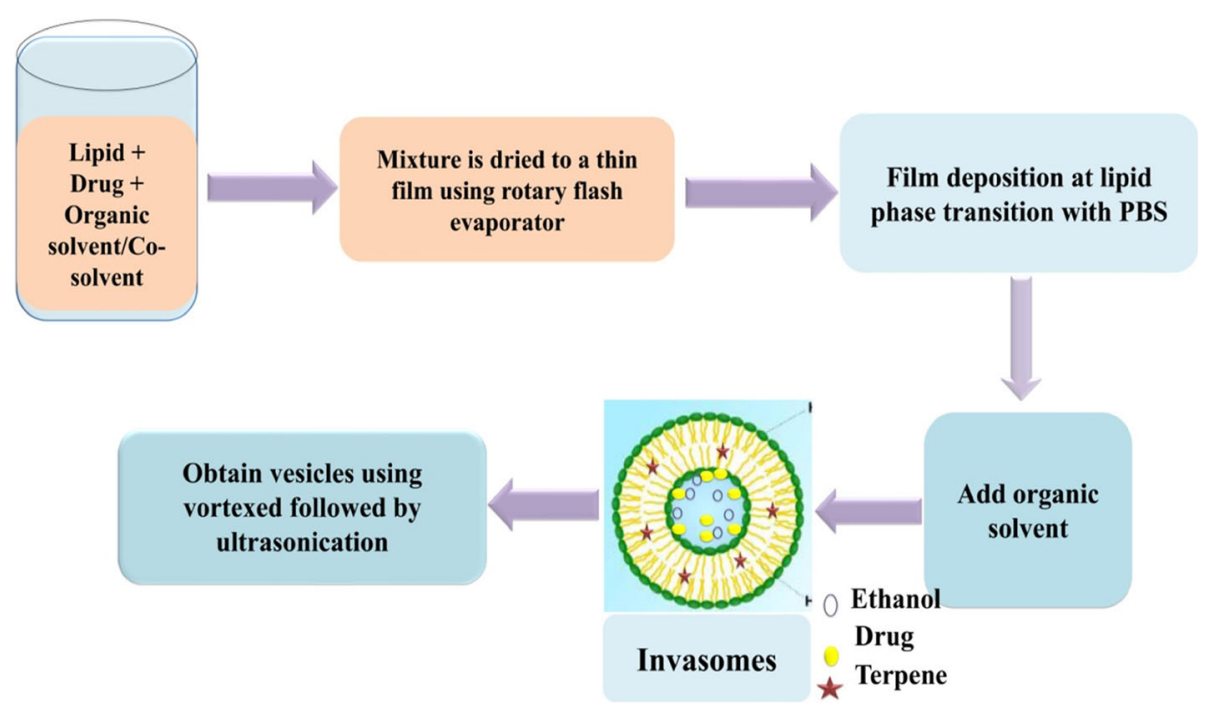

Fig. 4 Preparation of invasomes using film hydration technique 

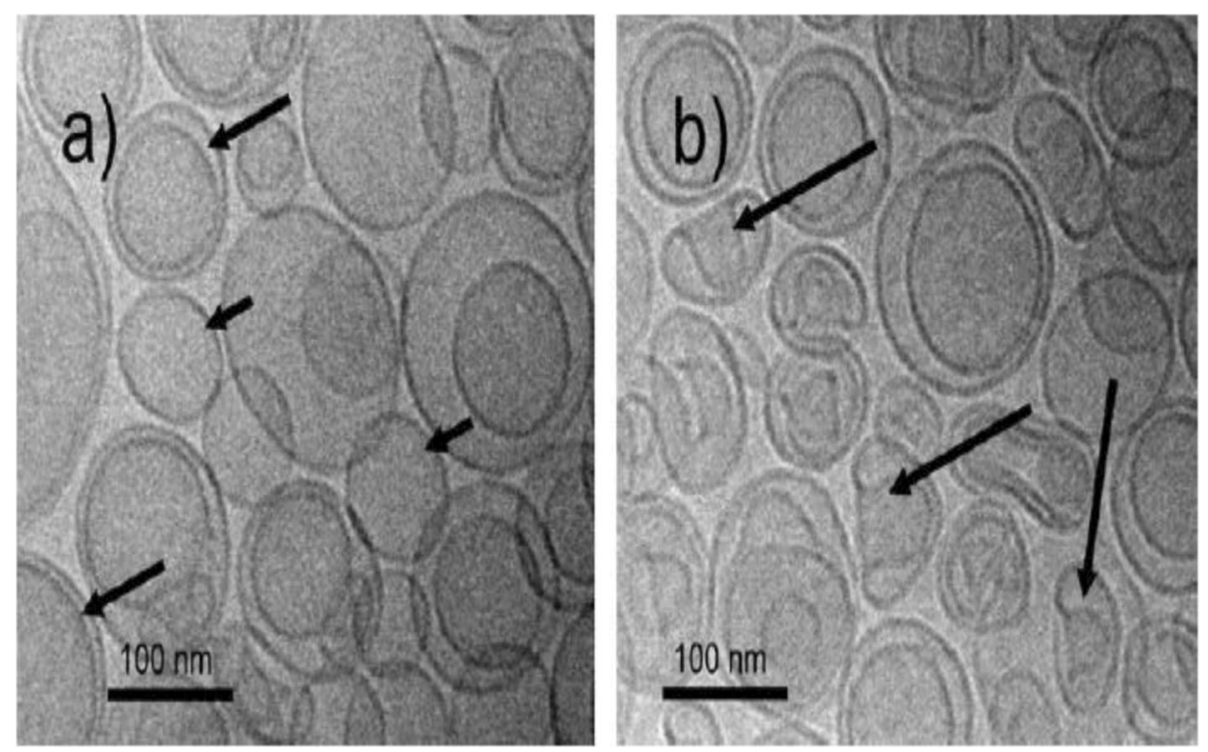

Fig. 5 Cryo-electron micrographs of temoprofin invasomes with a 1\% penetration enhancer mixture (1\% w/v citral). a Unilamellar (short arrows) and bilamellar (arrows of medium length) invasomes. b Long arrows represent deformed vesicles. (Reprinted from [33]. Copyright (2008), with permission from Elsevier.)

\section{Drug entrapment}

The drug entrapment efficiency is a necessary evaluation parameter in the drug delivery system. We can measure invasomal drug entrapment efficiency through many techniques such as ultracentrifugation techniques [26]. Lakshmi et al. have been reported that the entrapment efficiency of invasome varied with the types of terpene being used in the invasomal formulation. Besides this, the curcumin invasome entrapment efficiency based on types of terpene was declared to be limonene $>$ fenchone $>$ nerolidol. In this, they revealed that limonene has the highest entrapment efficiency because of a hydrocarbon group [43]. Whereas they accounted for the finasteride invasome for a maximum entrapment with limonene as compared with the nerolidol. Besides this, the $0.5 \%$ concentration of limonene exhibited high entrapment as compared with the $1 \%$ limonene concentration [30]. Moreover, we can increase the entrapment efficiency with the lipophilicity of the drug [39]. Consequently, the entrapment efficiency of invasomal formulation can be influenced by terpene concentration, terpene types, and drug lipophilicity.

\section{Drug content}

The drug content of formulated drug-loaded invasomes can be worked out and quantified using an ultraviolet spectrophotometer [40], modified high-performance liquid chromatography, etc. [2, 36].

\section{Skin permeation studies}

As per the literature survey, various types of skin such as human (female) abdominal skin obtained after plastic surgery [42], albino (male) Wistar rat skin [40, 41], porcine skin, rabbit skin after the removal of the fatty tissue [41] have been used for permeation studies. Also, Franz diffusion cells were used for the diffusion studies and the receptor compartment contained pH 7.4 PBS [40]. The confocal laser scanning microscopy can determine the capability of invasomal formulation to penetrate the skin [32]. Fascinatingly, such studies confirmed that nano-invasomes can easily penetrate the skin [40]. Moreover, the invasomes can combine with the lipids of the skin and altered the distribution of SC that loosens the tight lipid junctions [27]. Authors obtained the highest amount of temoprofin penetration using $1 \% \mathrm{w} / \mathrm{v}$ citral based invasomes as compared with the cineole and mixture of both [15]. Whereas, tolterodine tartrate invasomes containing 1\% terpenes revealed an enhanced penetration and accumulation in the skin as compared with the vesicles without terpenes [41].

\section{Stability studies}

The stability of invasomal dispersion, gel, suspension can be estimated by measuring the change in invasome vesicle size, PDI using DLS. On the account of stability study, we can ensure the shape of the invasomal formulation using SEM, TEM, or other appropriate techniques. The particle-containing charge changes can be worked out by evaluating the zeta potential of invasomal vesicles [2]. Initially, the finasteride invasomes were subjected to the stability study at $4^{\circ} \mathrm{C}$ and $25^{\circ} \mathrm{C}$ temperatures for 120 days. After 120 days it was observed that the zeta potential and entrapment efficiency of vesicles stored at $4{ }^{\circ} \mathrm{C}$ 
did not vary much as related to the initial evaluation, but in fact of $25^{\circ} \mathrm{C}$, the variation in the result was considerable. Briefly, owing to a reduction in negative surface charge, the aggregation of invasome vesicles led to a decline in entrapped efficiency occurring in the physical instability of invasome vesicles stored at $25^{\circ} \mathrm{C}$. Therefore, it was pointed out that $4{ }^{\circ} \mathrm{C}$ is the optimum temperature for the storage of invasomes [30]. The stability profile of tolterodine tartrate invasome reported that to avoid the substantial loss of drug, the formulation should be stored at refrigerated temperature $\left(4 \pm 2{ }^{\circ} \mathrm{C}\right)$ instead of $30^{\circ} \mathrm{C}$ storage condition [41]. To sum up, the invasomal formulation can be more stable when it stores in the refrigeration condition.

\section{Penetration enhancement mechanism}

Plentiful literature verified the invasomes superior behavior compared with typical lipidic vesicles. Here, invasomes constituents i.e. terpenes, ethanols, phospholipids have shown to be very strong penetration enhancers. Concerning phospholipids, invasomes components containing phosphatidylcholine, a strong penetrator thanks its head group, which has a strong enhancing effect. Besides, phosphatidylcholine containing unsaturated acyl chains helps to improve the penetration ability of phospholipids [27, 46, 47]. Ample literature reported that the invasome vesicles improve the skin permeation of hydrophilic and lipophilic actives. In that, the credible mechanism involved in the permeation of the invasome from the surface of application to the object zone are further important for the delivery of active (Fig. 6). Interestingly, a combination of ethanol, terpenes, lipids resulted in a synergistic effect and accordingly enhanced the drug permeation through the skin [48]. During the application of invasomes or invasomal formulation on the skin initially, the fusion of a few invasomes with skin responsible for the fractional release of ethanol, eugenol (terpene), and active. Such an initial release of active on the skin surface may promote the local application over the membrane [27, 46, 47]. The SC lipids could fluidize with the release of small amounts of ethanol from invasive dispersion (being outside vesicles). Besides, a large part of the invasomes is likely fractured to penetrate the upper SC layers, leading to terpenes, ethanol, and unsaturated phospholipids being released (Fig 6a). That would go along with the findings of many authors who say vesicles disintegrate on the skin surface and molecular penetration of vesicle components (i.e. phospholipids) into the intercellary lipid matrix, mixing with the SC's intercellular lipid to change lipid layers and increase the drug penetration. The releases of ethanol, terpenes, and unsaturated phospholipids will also have free effects on penetration [27, 46-48]. Such penetration improvements are suggested for the fluidization of intercellular SC lipids as they all function through this mechanism. This could contribute to the development of microcavities and an increase in the free diffusion volume that could further enhance the spreading coefficient of the drugs released from the vesicle. Furthermore, phospholipids, ethanol, and terpenes also increase the partitioning of the substance into the intercellular lipid bilayer of the SC. However, because invasomes contain powerful penetrating agents, they often penetration function through other mechanisms. As regards the penetration of invasome vesicles, a large number of hypotheses are included that could make the permeation of small intact

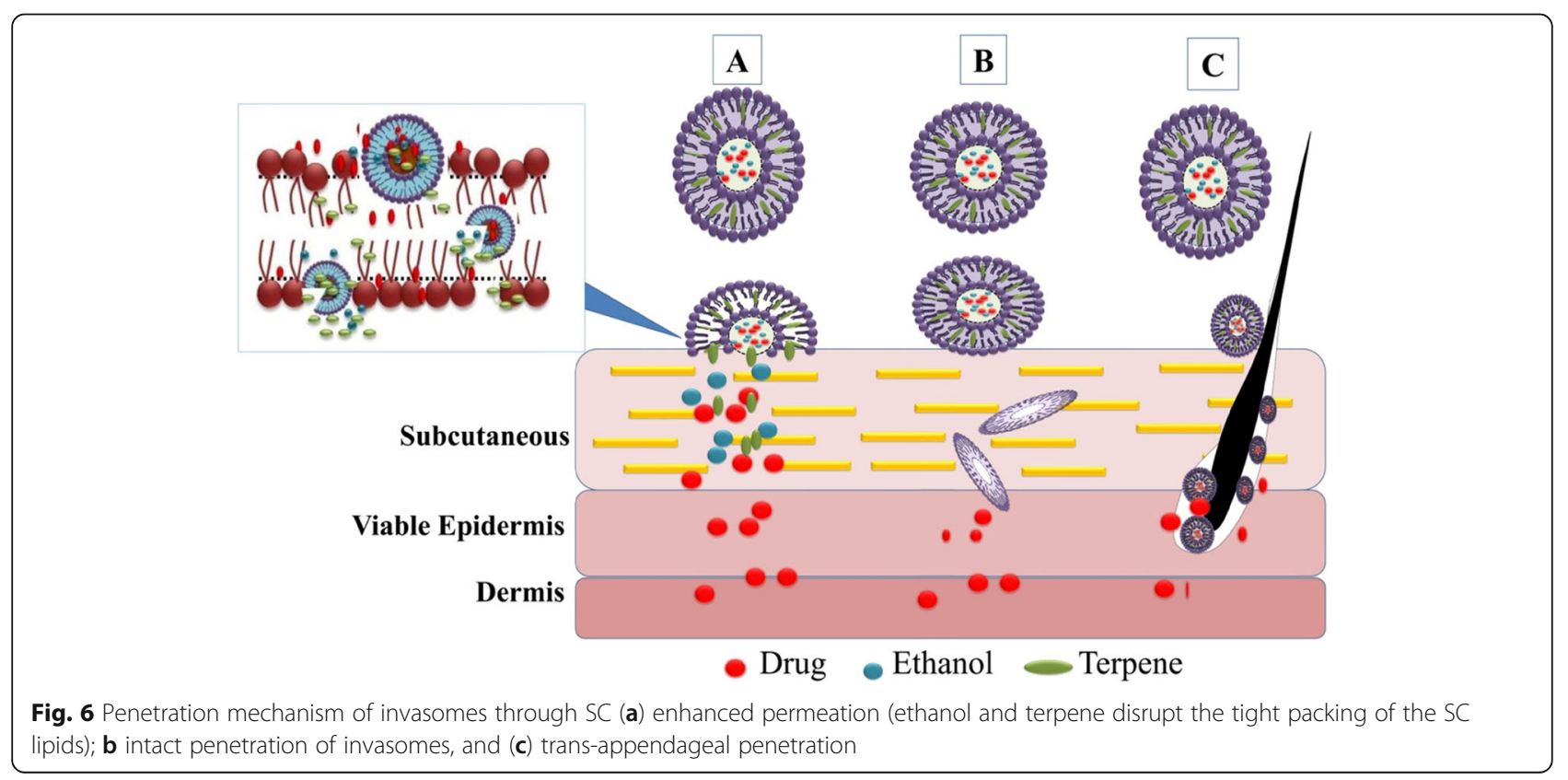


invasomes into SC. It involves disrupted SC lipid organization, high invasome vesicle fluidity due to the effect of ethanol and authorized terpenes. Furthermore, invasomes achieved a superior penetration rate, it may due to the high deformability of invasomes, that assumed due to the correlation between vesicles' high fluidity and deformability. The smaller vesicle size of the invasome also helps the penetration of the drug. Additionally, the existence of the transepidermal osmotic gradient is critical (driving force) to the diffusion of intact deformable, high hydrophilic vesicles, as they appear to obey the skin hydration gradient. On the other hand, the release of the drug into the skin layers can be a consequence of vesicles being fused into the intercellular lipids of the SC. Moreover, a significant route of invasomes penetration into the skin tended to be the pilosebaceous units (Fig 6c). For the anesthetic nature of terpenes, the released terpenes are expected to counter the sense of pain [27, 46-48]. In conclusion, the vesicle containing ethanol and eugenol increases the diffusivity, which contributes to a higher penetration rate of invasomes. The complete invasome vesicles can reach slowly to the pilosebaceous region via disturbing into the matrix of a lipidic layer of skin to get access to the deep dermal region. Besides the wide pores around the follicular unit serve as a canal for unbroken invasomes to facilitate localization in the target region via hair follicle as a supporting wick $[27,49]$.

\section{Pharmaceutical applications of invasomes Immunosuppressive drug delivery}

Immunosuppression is the primary approach to treating autoimmune diseases. Also, it is useful for the clinical application of existing immunosuppressive agents that have been suffered from various drug side effects. The nanotechnology centered approaches can correct the major limitations by enhancing immunosuppressant delivery to target cells of the immune system. Also, reducing the recommended dose for therapeutic function and reducing drug distribution to non-target tissues can be a key alternative to immunosuppressive drug delivery [50]. From the sub-structure of lipidic vesicles in drug delivery, it receives the primary consideration of the investigators to develop advanced nanosized vesicles. A literature survey mentioned that cyclosporine A (CsA, CyA) is a lipophilic drug and it exhibits poor penetration efficiency into the skin layers (partition coefficient: 4000). The topical applications of CsA can be a suitable alternative for the management of psoriasis and other dermatological diseases. Initially, Verma were synthesized the invasomes nanocarrier for delivery of CsA, CyA using unsaturated soybean phosphatidylcholine $(10 \% \mathrm{w} / \mathrm{v})$, ethanol $(3.3 \% \mathrm{w} / \mathrm{v})$, citral:cineole:D-limonene mixtures $(0.5: 1.0: 1.5 \% \mathrm{w} / \mathrm{v})$ and PBS up to $100 \% \mathrm{w} / \mathrm{v}$ using mechanical dispersion. In vitro penetration study concluded that due to the presence of ethanol and terpenes, prepared invasome vesicles showed a higher amount of CsA in the deeper layer of skin (viable epidermis and dermis) as compared with the aqueous/ ethanolic drug solution and liposomes (without ethanol and terpenes). Besides, the increasing concentration of terpene $(0.5$ to $1.5 \%)$ significantly increased the amount of CsA in the deeper skin layer and subcutaneous layer. It shows the direct correlation between the amount of drug added into the terpene mixture and the amount of drug found in the skin layer [12].. Owing to excellent findings of immunosuppressive agent-loaded invasomes, it can be used for treating autoimmune diseases. Taken as a whole, it confirmed that the invasomes can be an effective substituent for hydrophobic drug delivery to the deeper skin layers.

\section{Anticancer drug delivery}

From its inception, cancer treatment is still a challenging field in the era of biomedical science. Due to the ineffectiveness of currently engaged therapeutic strategies, a large number of deaths have been occurring each year [51]. Therefore, there is an urge to develop an advanced substitute to resolve cancer ineffective treatment issues. Interestingly, the temoporfin is a potent (second-generation) photosensitizer. It shows high tumor selectivity and residual photosensitivity of only 2 weeks. Thus, this could be an effective anticancer agent in photodynamic therapy of early or recurrent carcinomas. On this account, Dragicevic-Curic and co-authors were reported that the deposition of the highly hydrophobic photosensitizer (temoporfin) using invasomes into the skin layer (SC). Briefly, temoporfin-loaded invasomes have been synthesized using the mechanical dispersion method. In that, the temoporfin and $1 \% \mathrm{w} / \mathrm{v}$ terpene (limonene/cit$\mathrm{ral} /$ cineole) were dissolved in ethanolic phospholipid solution (phosphatidylcholine:ethanol: 75:25 w/w) and subjected to vertexing followed by sonication for $5 \mathrm{~min}$. Finally, PBS was added into the above-mentioned clear solution with constant vortexing for $5 \mathrm{~min}$. The drugloaded invasome (1\%, w/v cineol) showed about 105.4 $\mathrm{nm}$ particle sizes and about 0.066 PDI. After plastic surgery, the human female abdominal skin was obtained and penetration was carried out using the nominal surface of the Franz cells $\left(3.14 \mathrm{~cm}^{2}\right)$. The use of cineole $(1 \%$ $\mathrm{w} / \mathrm{v})$ showed the highest penetration ability followed by a mixture $(1 \% \mathrm{w} / \mathrm{v})$ of cineole: citral: D-limonene $(45: 45$ : $10 \mathrm{v} / \mathrm{v})$. Experimental outcomes revealed that the single terpene could make an efficient delivery of temoporfin and a combination of terpenes could lead to the synergistic effect of active penetration to the subcutaneous and deeper skin layers. Besides, the stability study indicated that the invasomes containing $1 \% \mathrm{w} / \mathrm{v}$ cineole 
showed a small increase in particle size and PDL value and can be considered a physically stable form for 12 months. In the future, invasome can be an efficient carrier for the delivery of hydrophobic active molecules with effective concentration to the systemic/local site. However, there is no thumb rule for the use of terpene and its mixture towards penetration [15]. As abovementioned, temoporfin is a second-generation synthetic photosensitizer used for the treatment of oral carcinomas, refractory oral carcinomas, non-melanomatous tumors of the skin of the neck, and head. For photodynamic therapy, there is a need for sufficient skin penetration of photosensitizer. Dragicevic-Curic et al. reported that the invasome can promote the efficient delivery of temoporfin drug. In this context, they have prepared the temoprofin-loaded invasomes via mechanical dispersion method. In brief, temoporfin and $1 \% \mathrm{w} / \mathrm{v}$ of terpenes mixture [cineole, citral and D-limonene] were dissolved in ethanolic phospholipid solution (phosphatidylcholine:ethanol;75:25 w/w). Further, the mixture was subjected to vortexing and sonication to obtain a clear solution. After that PBS was added followed by constant stirring $(5 \mathrm{~min})$ to obtain multilamellar vesicles and then extruded using an Avestin hand-extruder via polycarbonate membranes $(400 \mathrm{~nm}, 200 \mathrm{~nm}, 100 \mathrm{~nm}, 50$ $\mathrm{nm}$ ). The particle size and PDI of invasomes (containing $1 \%$ citral) were found to $129.5 \mathrm{~nm}$ and 0.087 respectively. Furthermore, the use of terpene mixture (citral, cineol, and limonene) provides a higher penetration efficacy of photosensitizer due to the synergistic penetration effect. Besides this, the drug-loaded invasome photodynamic efficacy was carried out through topical application onto the skin of mice (bearing the subcutaneously implanted human colorectal tumor HT29) followed by photoirradiation. The result revealed that the use of photodynamic therapy for temoporfin-loaded invasome formulation developed the significantly $(p<0.05)$ smaller size tumor HT29 as compared with the control (ice without any treatment and mice only photoirradiation) [33]. Hence, invasomal (with $1 \%$ terpene mixture) topical application opens the new door as promising tools for photodynamic therapy of psoriasis/superficial skin tumors. As we know, anastrozole is an efficient aromatase inhibitor for the management of postmenopausal women with breast cancer. However, anastrozole shows many side effects and extensive first-pass metabolism. In 2019, Vidya and coauthors have developed the anastrozole-loaded invasomes using the film hydration method and then converted into invasomal gel. In this study, the phospholipids have been dissolved in ethanol: chloroform $(2: 1, \mathrm{v} / \mathrm{v})$ mixture followed by drying to obtain thin film rotary flash evaporator. In that, the pressure was reduced from 500 to $1 \mathrm{mbar}$ at $45^{\circ} \mathrm{C}$ and finally placed at $1 \mathrm{mbar}$ for $24 \mathrm{~h}$. Finally obtained film was hydrated using PBS, ethanol, drug, and fenchone for $30 \mathrm{~min}$. The final invasomal vesicles were ultrasonicated and extruded using a polycarbonate membrane. Interestingly, a combination of anastrozole and fenchone has been shown high entrapment of the anastrozole. Besides, the high fencing concentration provides a low boiling point and lipophilicity and high concentration. Lowering the boiling point of terpene provides the weak cohesiveness of the molecules, and due to that, they can easily associate with lipids of SC and modifies the barrier property. The drug-loaded invasome particle size, PDI, and zeta potential were found to be $226.4 \mathrm{~nm}, 0.540$, and $-20.9 \mathrm{mV}$, respectively. Due to the presence of ethanol in the sample, the negative zeta potential was found on the invasome vesicle, which helps to prevent the vesicle aggregation due to exhibited electrostatic repulsion. It would be more beneficial for the ensuring stability of invasomal dispersion. The ex vivo permeation and skin deposition of invasomes on male Wistar rat skin showed superior permeation, enhanced transdermal flux, and 73\% skin deposition of drugs. The repulsion forces of lipophilic vesicles and the hydrophilic nature of terpene resulted in the enhancement of percent entrapment, penetration, and accumulation rate of actives as a compared drug without vesicle carrier. The cytotoxic study on the Michigan Cancer Foundation MCF-7 cancer cell line showed the cytotoxic effect of optimized formulation (at $5 \mu \mathrm{L} / \mathrm{mL}$ ) [34]. These findings indicate that the characteristic of anastrozole skin deposition can be improved with invasomes to targeted drug action in the treatment of breast cancer in postmenopausal women to resolve the problem of oral administration of the active. Hence, it can pave the pathway for breast cancer treatment in postmenopausal women using anastrozole invasomal gel.

\section{Delivery of vitamin analog}

Isotretinoin is a vitamin A analog and used to treat eosinophilic pustular folliculitis. In recent attempts, Dwivedi and co-investigators have been revealed the synthesis of isotretinoin invasome using the mechanical dispersion method. In brief, isotretinoin dissolved eugenol was added into ethanolic egg lecithin and subjected to vortexing (60 min) which gives the homogeneous suspension. After that, hydration of vesicles has been performed using PBS $(\mathrm{pH}$ 6.8), which provides the yellowish translucent invasomes containing suspension. Finally, this suspension has been shifted to sonication (3 cycles/15 min), which provides excellent invasomes. The free isotretinoin has been separated from invasomes using a dialysis bag. In this study, the critical determinant revealed that the various factors of formulation affected the penetration rate of invasomes. The particle size, PDI, zeta potential, and entrapment efficiency of the optimized batch was found to be $148 \mathrm{~nm}$, $0.16,-69.2 \mathrm{mV}$, and $85.78 \%$, respectively. Amusingly, the increase in the concentration of egg lecithin increased the 
size of invasomes. On the other hand, the concentration of eugenol does not affect the vesicle size of invasomes. At a higher concentration of egg lecithin, the single hydrophobic chain with a polar head group of egg lecithin resulted in the highly positive curvature in membranes, which increased the invasome vesicle size. Besides this, the more lipid concentration increased the entrapment efficiency of the isotretinoin, because of the more lipids available to entrap the isotretinoin. In addition to this, the use of eugenol increased the solubility and the entrapment of isotretinoin. Similarly, the high concentration of eugenol and lecithin showed high deformability of invasome vesicles. Finally, the ex vivo permeation on rat-shaved skin has been carried out using a Franz diffusion cell. It showed 85.94\% cumulative isotretinoin permeation and about $78.82 \mu \mathrm{g} / \mathrm{h} / \mathrm{cm}^{2}$ topical flux $\left(J_{\max }\right)$. The prepared invasome gel showed $97.12 \%$ drug content and zero-order release with a 1.135 diffusion constant. Also, it accomplished $85.21 \%$ of cumulative isotretinoin permeation within $8 \mathrm{~h}$. Furthermore, invasomes gel arrested the cell growth up to $82 \%$ with an insignificant difference [27]. Overall, invasome gel would be capable of delivering the isotretinoin to the follicular unit and accordingly to achieve pilosebaceous targeting. In the future point of view, clinical trials (phases I, II, and III) will be necessary before the use of human patients.

\section{Used in alopecia treatment}

There is a tremendous need for progress in engaged therapies to efficient alopecia treatment. The literature says that the use of invasomes yields long-term effects that are self-satisfactory. Today, patients also use alternative and complementary therapies to try to find a safe, natural, and efficient cure for hair restoration [52]. In this context, the FDA approved the finasteride, a $5 \alpha$ reductase inhibitor, and now this is commonly preferred for the treatment of alopecia. Despite this, there are needs to develop a novel carrier for the delivery of finasteride across the dermis layer. Herein authors have been prepared the invasome of finasteride using the combination of a terpene (limonene, nerolidol, and carvone: $0.5 \%, 1.5 \%$, and $1 \%$, accordingly) through mechanical dispersion. In brief, soya phosphatidylcholine $(10 \% \mathrm{w} / \mathrm{v})$ was added into ethanol $(40 \% \mathrm{w} / \mathrm{v})$ and vortexed $(5 \mathrm{~min})$ to obtain the ethanolic lipid mixture. After that, the finasteride $(0.35 \% \mathrm{w} / \mathrm{v})$ has been added into terpene with different concentrations $(0 .-1.5 \% \mathrm{w} / \mathrm{v})$ followed by constant vortexing and sonication ( $5 \mathrm{~min}$ ) to which gives the clear solution. the hydration of vesicles has been achieved using PBS (up to $100 \% \mathrm{w} / \mathrm{v}$ ) with constant stirring (5 min). Finally, drug-loaded multilamellar invasome vesicles were subjected to probe sonication $\left(5\right.$ cycles $/ 5 \mathrm{~min}$ ) at $4{ }^{\circ} \mathrm{C}$. The $0.5 \%$ limonene containing optimized invasomes showed about $84.56 \%$ of entrapment efficiency, $-69.1 \mathrm{mV}$ of zeta potential, $5.81 \mu \mathrm{m}$ of particle size, and $5.94 \mu \mathrm{g} / \mathrm{cm}^{2}$ of cumulative amount permeated. Herein, finasteride-loaded invasomes showed a negative charge on the surface of vesicles due to the presence of ethanol. Besides, it resulted in electrostatic repulsion, which provides better stability to the invasomal dosage form. The sonication of invasome gives the high rotation energy that results in a high negative charge/potential. Besides that, ethanol concentration showed a negligible change in the negative charge with different terpene phases and resulted in the progress of the finasteride penetration rate. The concentration of terpene and entrapment efficiency was found to be inversely proportional to each other. Concisely, hydrophobic limonene showed the maximum entrapment ability of finasteride. On the other hand, hydrophilic nerolidol showed the minimum entrapment of finasteride. Owing to the presence of hydrocarbon and ketone group in limonene and carvone which gives the more hydrophobic nature, provides more entrapment of actives. Furthermore, limonene, nerolidol, and carvone act by lipid extraction of SC increasing the diffusion coefficient of active and disruption of highly ordered intercellular lipid structure of SC, respectively. The in vivo study of transdermal invasomal gel using a rabbit model exhibited a maximum concentration of $656.53 \pm 25.03 \mathrm{ng} / \mathrm{mL}$. Besides this, the $\mathrm{AUC}_{0-72}$ was 3.04 times higher than that of the oral route (5630.58 \pm $361.50 \mathrm{ng} \mathrm{h} / \mathrm{ml}$ ). Additionally, the bioavailability of finasteride was enhanced by $303 \%$ using invasomes. The use of the current invasomal drug-loaded formulation demonstrated hemorrhage, edema, congestion, infiltration of mononuclear cells, thickening of epidermis degeneration, and fatty changes in the dermis as compared with the well-woven structures with distinct SC in control. In conclusion, the iontophoresis of invasome formulation revealed the enhancement in the penetration rate of finasteride [30]. Hence, in the future, these new lipid vesicles can be alternative carriers for iontophoretic transdermal delivery of finasteride. The minoxidilloaded invasome formulation using soya lecithin and different penetration enhancers via film hydration method reported in 2009 by Mura et al. In this pioneered study, three penetration enhancers namely transcutol ${ }^{\circ}$, labrasol ${ }^{\circ}$, and cineole have been used. Out of this, cineole and transcutol are well-known nontoxic, biocompatible penetration enhancers, whereas labrasol is a safe, non-ionic hydrophilic surfactant. Briefly, soya lecithin, minoxidil, dicetylphosphate, and penetration enhancers have been dissolved into the chloroform. After that, the lipid-minoxidil mixture has been deposited as a thin film using a roto-evaporator. After the removal of chloroform, the hydration has been done using distilled water under constant mechanical stirring at room temperature. Then, vesicle suspension was subjected to sonication and after that 
freeze-drying $\left(-20{ }^{\circ} \mathrm{C}\right.$ for $12 \mathrm{~h}$ ). As compared with the average diameter of cineole vesicle $(144 \mathrm{~nm})$, labrasol $(202 \mathrm{~nm})$, and transcutol $(171 \mathrm{~nm})$ showed a larger diameter. It may due to hydrophilic molecules increase vesicle surface energy and that shows vesicle enlargement. Also, it exhibited homogeneous and monodisperse suspension with an excellent PDI < 0.3, which confirms the stability of invasomes formulation. Furthermore, it showed negative zeta potential (from - 52 to $-58 \mathrm{mV}$ ), which ensured the prevention of vesicle aggregation. Furthermore, an in vitro diffusion study using newborn pigskin showed a statistically significant improvement of minoxidil deposition in the upper skin in comparison with drug liposomes and drug ethanolic solutions. Additionally, it avoids the transdermal delivery of minoxidil which helps to improve the cutaneous minoxidil bioavailability [35]. Therefore, the use of penetration enhancers improved deformable vesicles than conventional ones. In vitro penetration revealed that the use of penetration enhancers increases the deposition of minoxidil into the skin membrane than the alcoholic solution of minoxidil. Therefore, it can be a potential innovative carrier for improving the specific topical delivery and accordingly bioavailability of minoxidil.

\section{Delivery of anti-acne agent}

Acne is a widespread skin disorder worldwide in recent times [53]. Dapsone is an exceptionally active pharmaceutical ingredient for leprosy treatment. It has significant potential for acne therapy due to its antiinflammatory action. As essential for the delivery of topical drugs, an effective carrier must be established for the delivery of dapsone to the specific site. In consequence, El-nabarawi et al. prepared the dapsone-loaded invasome by a film hydration technique using terpene (limonene, cineole, citral, or fenchone) and phosphatidylcholine for the treatment of mild to moderate acne. Briefly, dapsone $(20 \mathrm{mg}$ ) and terpenes with different concentrations were mixed with a clear solution of phosphatidylcholine $(200 \mathrm{mg})$ in methanol/chloroform, 2:1 v/ v. After that, this mixture was subjected to rotary evaporation to remove the organic solvent at $120 \mathrm{rpm}\left(60{ }^{\circ} \mathrm{C}\right)$ for $15 \mathrm{~min}$. Finally, thin-film was hydrated using $3 \% \mathrm{v} / \mathrm{v}$ ethanolic: water mixture at $120 \mathrm{rpm}\left(60{ }^{\circ} \mathrm{C}\right)$ for $1 \mathrm{~h}$ and further filtered (pore size $25 \mu \mathrm{m}$ ) to separate the drug crystals from invasomes. The high concentration of terpene showed the superior entrapment efficiency of dapsone. Furthermore, the limonene containing invasomes showed the highest entrapment efficiency followed by cineole, fenchone, and citral. Possibly, it may because of the lipophilicity of the used terpenes. The optimized drug-loaded unilamellar invasome exhibited uniform spherical discreet shape, and $-37.5 \mathrm{mV}$ zeta potential that confirms the stability of invasome vesicles. The differential scanning thermogram revealed that the invasomecontaining drug was converted into the amorphous region with uniform distribution. Interestingly, the in vivo permeation study on Wistar rats resulted in the enhancement of penetration rate of invasome by 2.5 -fold as compared with the solution form. Besides, they found the in vivo rat skin deposition amount of dapsone to be $4.11 \mu \mathrm{g} / \mathrm{cm}^{2}$ for invasomes, which was high than the drug-alcoholic solution $\left(1.71 \mu \mathrm{g} / \mathrm{cm}^{2}\right)$. Particularly, the Wister albino rats have been shown invasome increases the 2-fold $\mathrm{AUC}_{0-10}$ than the solution of dapsone [36]. Overall, invasomes significantly increased the skin deposition of dapsone. Therefore, invasome can be potential vehicles for the delivery of an anti-acne agent. A literature survey reported that capsaicin is a major pungent photo component and widely studied in the pharmaceutical arena. Particularly, it is used for the management of oral and topical pain. In 2016, Duangjit et al. synthesized capsaicin (0.15\%) loaded invasomal formulation using phosphatidylcholine and D-limonene. The optimized capsaicin-loaded invasomes showed narrow size distribution (0.01-0.30), smaller than $100 \mathrm{~nm}$ in size. Furthermore, negative $(-20 \mathrm{mV})$ zeta potential confirmed the stability of drug-loaded invasomes. Furthermore, it gives excellent skin permeability over the conventional liposome formulation and commercial product (0.15\% capsaicin in ethanolic solution) [37]. Hence, the optimized capsaicin invasomes can be used as a substitute for transdermal drug delivery.

\section{Tyrosine's inhibitors}

Phenyl ethyl resorcinol topical administration requires further focus because of its strong anti-tyrosinase activity and a skin-lightening agent. Elastic vesicles are urged to evolve and can solve many problems related to phenyl ethyl resorcinol such as solubility, flexibility, skin irritation, and permeation. Therefore, in 2018, Amnuaikit and co-authors have developed the phenyl ethyl resorcinol loaded invasomes using terpene ( $1 \% \mathrm{w} / \mathrm{v}$ D-limonene) with absolute alcohol $(10 \% \mathrm{v} / \mathrm{v})$ and $15 \% \mathrm{w} / \mathrm{w}$ sodium deoxycholate as an edge activator through film hydration method. In brief, phenyl ethyl resorcinol invasomes contain the different terpene (fenchone, citral, and D-limonene) with $10 \%(\mathrm{v} / \mathrm{v})$ ethanol as a skin penetration enhancer. After that, a hydro-ethanolic solution consisting of water and absolute ethanol $10 \%(\mathrm{v} / \mathrm{v})$ was used as an aqueous phase. After that, to obtain a homogeneous solution, the oil phase and aqueous phase were separately sonicated at $60^{\circ} \mathrm{C}(30 \mathrm{~min})$. Then, the organic solvent (absolute ethanol) was removed from the oil phase via rotary evaporation followed by film hydration using an aqueous phase with normal shaking (5 min). Finally, the invasome vesicles were sonicated $30 \mathrm{~min}$ at $60{ }^{\circ} \mathrm{C}$ to obtain the complete invasomes. These drug-loaded 
invasomes resulted in $<500-\mathrm{nm}$ vesicle size, $<0.3 \mathrm{PDI}$, high zeta potential, $>50 \%$ entrapment efficiency, and good stability at $30{ }^{\circ} \mathrm{C}$ at $75 \% \mathrm{RH}$ (4 months). Furthermore, in vitro phenyl-ethyl-resorcinol accumulation in full-thickness newborn pig skin demonstrated that the application of elastic carrier formulations significantly attends higher accumulation of phenyl ethyl resorcinol as compared with liposomes. The in vitro permeation study showed $85.38 \%$ recovery, which is close to the acceptable limit (90 to 110\%). Due to the SC properties, the low penetration of phenyl ethyl resorcinol has resulted. Besides, phenyl ethyl resorcinol invasomes showed up to $80 \%$ anti-tyrosinase activity and through an acute irritation test in rabbits. It confirmed the safe skin application of phenyl ethyl resorcinol invasomal formulation $(0.5 \% \mathrm{w} / \mathrm{v})$. The prepared invasomes and transfersomes showed superior tyrosine's inhibition activity and reduction of melanin in B16 melanoma cells as compared with the conventional liposomes [38]. Consequently, invasomes presented suitability for phenyl ethyl resorcinol delivery, which is highly effective for skin lightening products.

\section{Treatment of erectile dysfunction}

Erectile dysfunction is an inability to start or maintain the required penile erection during satisfying sexual intercourse and millions of men's population are affected by erectile dysfunction. Whereas about 30 million of the men's population has been added to the erectile dysfunction each year, and only 2 lakhs men pursue treatment from a physician. Therefore, there is an urge to investigators from the suffered population to treat erectile dysfunction. Owing to this, there is a need to deliver the drug efficiently using a suitable carrier which can overcome the limitations of previously engaged techniques [54]. Avanafil is a selective phosphodiesterase type 5 inhibitor (FDA approved), generally used for oral administration in the treatment of erectile dysfunction. Although, the oral bioavailability of the avanafil is challenging due to poor aqueous solubility, extensive presystemic metabolism. Additionally, there is a chance of alteration of the absorption of drugs in the presence of food. In 2019, Ahmed et al. were prepared the avanafil-loaded invasomes for the treatment of erectile dysfunction using Phospholipon ${ }^{\circ}$ 90G, D-limonene via film hydration method. Briefly, avanafil-loaded invasomes have been synthesized by dissolving avanafil $(100 \mathrm{mg})$ and Phospholipon ${ }^{\circ}$ 90G in methanol/chloroform mixture $(1: 2 \mathrm{v} / \mathrm{v})$ followed by removal of organic solvent using rotary evaporation. The deposited avanafil lipid films have been kept in a vacuum cabinet to remove the organic solvent overnight. Further, film hydration has been carried out using PBS/ethanol mixture $(7: 3)$ in rota vapor at $60 \mathrm{rpm}\left(25^{\circ} \mathrm{C}\right)$ for $1 \mathrm{~h}$ and then shifted to sonication in an ice bath to obtain nanosized invasome vesicles. The optimized avanafilloaded invasome vesicles (10.47\% phospholipid, $2.00 \%$ ethanol, and $1.50 \%$ D-limonene) resulted in about $96.98 \%$ entrapment efficiency. Moreover, the vesicular size and PDI of invasomes was found to be $109.92 \mathrm{~nm}$ and $<0.3$, respectively. Interestingly, the PDI value confirmed the homogenous and mono-dispersedness of avanafil invasomes. Then, the optimized avanafil invasomal formulation has been further incorporated into the hydroxypropyl methyl cellulose-based transdermal film. They have reported that the change in the $\beta$-citronellol to D-limonene reduced the size of the invasome and it could be an effect of molecular weight and lipidic nature of terpene. Additionally, the $\beta$-citronellol has less lipophilicity than the Dlimonene, which increased the force among vesicles and terpene and therefore, caused the enlargement of invasome size. The exorbitant amount of lipids leads to an increase in the lipid particles forming each vesicle, and these raise the possibility of the drug to incorporate with a lipidic portion of the invasomes. The optimized AVA invasomal film enhanced ex vivo permeation with an enhancement factor of 2.514 which was performed on excised abdominal Wistar rat skin using a Franz diffusion cell and compared with avanafil raw film. The relative bioavailability of invasome was found to be more than 4-fold increased as compared with the raw avanafil-loaded film. The above mentioned findings of drug loaded invasomal transdermal film could be taken as a promising avanafil delivery method to resolve the enlisted problems such as poor aqueous solubility, extensive presystemic metabolism, etc., faced by the oral drug absorption [39]. Therefore, invasomal formulation provides the capability to enhance the skin permeation and bioavailability of avanafil. Consequently, it can be used as an innovative carrier for drug delivery in the treatment of erectile dysfunction.

\section{Delivery of anti-hypertensive agent}

Anti-hypertensive are used to treat elevated hypertension. It has numerous problems such as low aqueous solubility, low bioavailability, short biological half-life, low permeability, and a list of undesirable side effects [51]. This can be waved off using a suitable route for delivery and formulation. A calcium channel blocker, isradipine is generally used for the treatment of hypertension. Unfortunately, it has low oral bioavailability and it suffers the first-pass metabolism. Kamran et al. accomplished the development of invasome using Phospholipon ${ }^{\circ} 90 \mathrm{G}(2 \% \mathrm{w} / \mathrm{v})$, b-citronellene $(0.1 \% \mathrm{w} / \mathrm{v}$, terpene), and ethanol $10 \% \mathrm{w} / \mathrm{v}$ through conventional film hydration technique and used as an efficient carrier for the delivery of isradipine via the transdermal route. In brief, isradipine, terpene, and Phospholipon ${ }^{\circ}$ 90G were dissolved in chloroform: methanol $(2: 1 \mathrm{v} / \mathrm{v})$. Then, the organic solvents were removed through rotary evaporation 
and organic solvent traces were collected separately using a vacuum cabinet overnight. The hydration of isradipine invasomal lipid film has been performed using PBS: ethanol at $60 \mathrm{rpm}$ using rotary evaporator for $1 \mathrm{~h}$ and then subjected to probe sonification $\left(4^{\circ} \mathrm{C}\right)$ at $40 \%$ output frequency. The particle size, polydispersity index, entrapment efficiency, and transdermal flux through rat skin of isradipine invasomes were found to be $194 \mathrm{~nm}$, $0.272,88.46 \%$, and $22.80 \mathrm{mg} / \mathrm{cm}^{2} / \mathrm{h}$, respectively. Because of the presence of ethanol and terpene, it provides particle deformability and enhances the penetration rate of isradipine. Interestingly, enhancement in the deformability of the invasomes and lipidic bilayer of the SC disruption facilitates the penetration of isradipine invasome vesicles [26]. Hence, the prepared isradipine invasomes follows the transepidermal osmotic gradient into and through the skin SC and deliver the antihypertensive agent to the systemic circulation. In short, invasomes delivery systems could be a potential supplier of isradipine through transdermal for hypertension treatment. Abundant literature surveys reported that the hydrophobic olmesartan medoxomil exhibited low oral bioavailability and short biological half-life. It is preferably used to treat hypertension, but it also offers the aforementioned limits for oral administration of olmesartan. In 2016, Kamran et al. investigated nano-sized invasome for the delivery of olmesartan medoxomil through the transdermal route using the film hydration method. In brief, olmesartan (10 mg) and Phospholipon $90 \mathrm{G}$ mixture were dissolved into 1: $2 \mathrm{v} / \mathrm{v}$ of methanol: chloroform $(10 \mathrm{~mL})$. Then, thin layer lipid films have been obtained using rotary evaporation which removes the organic solvent when applied vacuum with appropriate time at a temperature more than lipid transition temperature. Moreover, the film hydration was carried out using PBS and ethanol that gives the multilamellar invasome vesicles. Finally, these obtained invasome vesicles have been subjected to obtain the nanosized invasomes. The vesicle's size, entrapment efficiency, and transdermal flux of invasomes were found to be $83.35 \mathrm{~nm}, 65.21 \%$, and $32.78 \mathrm{mg} / \mathrm{cm}^{2} /$ $\mathrm{h}$, respectively. In this study, the terpene (i.e., bcitronellene) has shown admirable entrapment and penetration capacity of olmesartan invasomes. The bcitronellene and phospholipid concentration are directly proportional to the size of invasomes and entrapment efficiency invasome vesicles, respectively. The nanosized invasomal gel showed a smooth, homogeneous appearance and texture and basic $\mathrm{pH}$ and exhibited no risk of skin irritation. Furthermore, the olmesartan release kinetics from invasomal gel follows the Higuchi matrix model $\left(R^{2}: 0.986\right)$, which confirmed that the invasomal gel exhibited the diffusion process-based olmesartan release. Moreover, the rat skin showed that the olmesartan invasome eventually distributed and permeated deep into the rat skin. Besides, olmesartan invasome improved bioavailability (1.15 times) in Wister rats [40]. It indicated that nano-invasomes were a competent transdermal delivery system for olmesartan. Thus, invasomes could be a more superior carrier for the delivery of other antihypertensive drugs via the transdermal route.

\section{Anticholinergic agent}

Tolterodine tartrate has extensive liver metabolism, aqueous solubility $>1 \mathrm{mg} / \mathrm{ml}$, molecular weight $<500 \mathrm{Da}$, low dose $<10 \mathrm{mg} /$ day, half-life $<10 \mathrm{~h}$, etc. which are ideally suitable characteristics for transdermal drug delivery. Besides this, oral administration of tolterodine tartrate having side effects such as dizziness, tachycardia, dry mouth, and gastrointestinal obstructive disorder. Hence, there is a necessity to overcome these major limitations, and deliver the tolterodine tartrate in a manner, which increases patient compliance, reduces the side effects. Additionally, it should maintain the drug plasma level constant for a specific time and can be terminated in serious conditions. In 2013, the tolterodine tartrateloaded invasomes have been synthesized using soy lecithin, ethanol, limonene, fenchone, and anethole through the film hydration method. In brief, phospholipid was dissolved in chloroform and slowly dried to a thin film through the rotary flash evaporator. They decrease the pressure between 500 and 1 mbar at a lipid transition temperature. Thus, the lipid film hydrated with a tolterodine tartrate containing a mixture of PSB (pH 7.4), 10\% $(\mathrm{v} / \mathrm{v})$ ethanol, and $1 \%(\mathrm{v} / \mathrm{v})$ terpenes for obtaining invasomes, at the lipid transition temperature for $30 \mathrm{~min}$. After that, the deposited lipid film was hydrated for 30 $\mathrm{min}$ at lipid transition temperature with a mixture of phosphate buffer ( $\mathrm{pH} 7.4$; SPB) containing drug, 10\% (v/ v) ethanol, $1 \%(\mathrm{v} / \mathrm{v})$ terpenes to obtain invasomes. Finally, tolterodine tartrate-loaded vesicles were subjected to ultrasonication which provides the nanosized tolterodine tartrate invasomes. Interestingly, tolterodine tartrate invasome vesicle size and PDI were found to be $1.3 \mu \mathrm{m}$ and 0.188 , respectively, which confirmed the uniform distribution of drug-loaded invasomes. Furthermore, the lecithin concentration (up to 3\%) was found to be directly proportional to the tolterodine tartrate entrapment in invasome vesicles. Also, invasomes containing limonene showed better permeation of tolterodine tartrate invasomes (limonene $>$ anethole $>$ fenchone) than the other terpene (fenchone and anethole). In addition to this, the prepared tolterodine tartrate invasomes shown admirable skin penetration as compared with pure drugs. Overall, terpene played a principal role in the percutaneous permeation (rabbit skin) of the tolterodine tartrate. They conclude that the highest lipophilicity of the terpene (limonene) shows the highest increase in the flux. Moreover, the smaller sized tolterodine tartrate 
invasomes may pass through small hydrophilic pores present in the intracellular space of the SC. The highly flexible tolterodine tartrate invasomes were formed due to the presence of terpenes and ethanol. Interestingly, the permeation order of tolterodine tartrate invasomes was found to be limonene $>$ anethole $>$ fenchone. Finally, the terpene with iontophoresis showed an increase in tolterodine tartrate flux. The negative zeta potential of invasomes and the iontophoretic drug transport demonstrated that the higher permeability of tolterodine tartrate-loaded invasomes as compared with the free tolterodine tartrate. Findings from this study have shown that the fusion of improved physical and chemical penetration techniques can accomplish additive amplificatio $\mathrm{n}[41]$. Therefore, a combination of physical and chemical penetration enhancing techniques can be a suitable substitute for transdermal delivery of anticholinergic agents and other active pharmaceutical ingredients.

\section{Antioxidant}

Nowadays, ferulic acid (antioxidant) is gaining much attention from research scholars due to its therapeutic effects such as anticancer, anti-skin disorders, antidiabetes, anti-cardiovascular disease, anti-inflammatory, etc. Ferulic acid is normally located in many of the plant cell walls. Unfortunately, it takes a short half-life of removal and needed many dosages with regular administration. In this regard, appropriate transdermal vesicles may also be a safer choice for ferulic acid delivery. Chen and co-investigators prepared the terpene (limonene, citral, cineole-1:4.5:4.5 v/v) based invasomes for the delivery of ferulic acid using the film hydration method and compared with conventional liposomes, ethosomes, Tween 80-based deformable liposomes. In brief, soybean phosphatidylcholine $(133 \mathrm{mg} / \mathrm{mL})$, ferulic acid $(12 \mathrm{mg} /$ $\mathrm{mL})$, and terpenes $(10 \mathrm{mg} / \mathrm{mL})$ were dissolved in methanol and chloroform mixture $(1: 2 \mathrm{v} / \mathrm{v})$ and then subject to removal of organic solvent using rotary evaporation under specified vacuum condition for the appropriate time at $43{ }^{\circ} \mathrm{C}$. The thin lipid film hydration was accomplished using PBS (pH 7.4) and ethanol (10\% v/v). Further, ferulic acid-loaded invasomes were subjected to sonication for $15 \mathrm{~min}$ ( $5 \mathrm{~min} /$ cycles $)$ in an ice-water bath that gives the nanosized invasomes. Finally, obtained invasomal suspension has been shifted to sized using polycarbonate membrane (pore diameter: $100 \mathrm{~nm}$ ), which gives the uniform ferulic acid invasomes. The ferulic acid invasomes exhibited about - 39-mV zeta potential, 129.1-nm vesicle size, and PDI $<0.2$. It confirmed that the developed suspension of ferulic acid invasomes had a stable, uniform, nanosized, and homogeneous form. In addition to this, the in vitro permeation of ferulic acid from ethosomes through the human skin was found to be high as compared with the other formulations. It may be because of the high concentration of ethanol in ethosomes. On the other hand, conventional liposomes have been expected to be effective for the delivery of drugs into the upper layers of the skin. Besides, ferulic acid invasomes showed better permeation because of deformable vesicles and penetration enhancer's interaction with lipid lamellae and skin layer [42]. In present pioneering attempts, Shah and co-authors investigated the penetration rate of antioxidant/anticancer and anti-acne agents, for example, idebenone and azelaic acid, respectively, in the original carrier systems (cationic LeciPlex, invasomes, and conventional liposomes). In brief, initially, idebenone/ azelaic acid was dissolved in $3 \mathrm{~mL}$ organic solvent (chloroform: methanol) and further mixed with an ethanolic solution of soybean phospholipid (75\%). After that, a thin lipid film was developed using rotary evaporation and then subjected to nitrogen drying $(15 \mathrm{~min})$. Finally, a mixture of terpenes, PBS, and ethanol with lipid film vortexed to obtain multilamellar invasome vesicles. The homogenous and uniform nanosized invasomes have been achieved using polycarbonate membranes $(100 \mathrm{~nm})$. The particle size, PDI, and entrapment efficiency of idebenone and azelaic acid invasome vesicles were found to be $140 \mathrm{~nm},<0.1$, and more than $90 \%$, respectively, which is much smaller than other formulations (cationic LeciPlex, and conventional liposomes). Particularly, single-step mixing without homogenization showed higher vesicle sizes and PDIs of LeciPlex formulations. In the case of drug-loaded invasomes, the existence of phosphatidylinositol and phosphatidic acid invasomes exhibited negative zeta potential $(-13 \mathrm{mV})$, which confirmed the stability of invasomes. Idebenone and azelaic acid invasomes were demonstrated in unilamellar vesicles along with a mixture of the small and large unilamellar vesicles, which confirmed that incorporating drugs into vesicles does not affect the vesicle shape. On the other hand, an ex vivo human skin penetration study revealed that the interaction of idebenone with the phospholipid membrane. Idebenone has been delivered by LeciPlex significantly high as compared with the invasomes. Besides this, the azelaic acid-based invasome showed a higher penetration ability for azelaic acid as compared with idebenone, while the interactions between carboxylic groups of the azelaic acid and the cationic surfactants resulted in low penetration of azelaic acid-loaded LeciPlex. Azelaic acid interacts with phospholipid containing phosphate and weak interaction with fatty acid chains. Due to the presence of phospholipid, the invasome exhibited in the L-lamellar phase and showed a high penetration ability [25]. In conclusion, for azelaic acid delivery invasome is a suitable carrier while for idebenone LaciPlex. This hypothesis was confirmed by the in vivo effectiveness analysis of azelaic acid formulations. Intracutaneous delivery of active pharmaceutical agents is developed based on pharmaceutical properties of drug, formulation variable characteristics, and formulation properties 
benefit of the entire. Therefore, during formulation development, the property of the drug, formulation, and its components has holds the major account.

\section{Miscellaneous applications of invasomes}

Curcumin is an active phytoconstituents obtained from Curcuma longa Lin. It has high potential in the treatment of various common and serious health issues such as hepatic disorders, hypocholesterolemic, anticoagulant, Alzheimer's disease, rheumatism, anorexia, anti-carcinogenic, anti-inflammatory, antispasmodic, antibacterial, etc. Nevertheless, curcumin is a poorly water-soluble active phytoconstituent and it has poor bioavailability. Owing to that several attempts have been made to overcome these issues using different innovative strategies. In 2014, Lakshmi et al. have been developed the curcumin-loaded cyclodextrin and hydroxypropyl $\beta$-cyclodextrin complex for solubility enhancement and then this complex loaded into the invasomes. On this account, various types of terpene with different concentrations (limonene, fenchone, nerolidol: 0.5, $1.0,1.5 \%)$ have been used as a penetration enhancer. In brief, the complexation of curcumin has been achieved using curcumin: cyclodextrin and hydroxypropyl $\beta$ cyclodextrin (1:2) through the co-precipitation method. Then, the curcumin complex was loaded into invasomes via mechanical dispersion using ethanolic solution soya phosphotidylcholine $(1-3 \% \mathrm{w} / \mathrm{v})$ followed by constant vortexing $(5 \mathrm{~min}$ ) with the addition of curcumin complex and terpenes. Then, the obtained solution has been subjected to sonication $(5 \mathrm{~min})$ and further hydration of lipid thin film has been achieved by adding PBS (10\% w/v) under constant vortexing ( $5 \mathrm{~min}$ ). Finally, it was converted to the gel form of invasomes. Interestingly, the vesicle size and PDI of curcumin invasomes were found to be $134.7 \mathrm{~nm}$ and $<0.2$, respectively. Moreover, due to the presence of ethanol, it showed negative zeta potential $(-33.7 \mathrm{mv})$, which confirm the stability of the invasomes. Owing to the high concentration of hydrophobic groups in limonene, invasome showed greater entrapment of curcumin. The entrapment efficiency of the curcumin complex with terpene was found in limonene $>$ fenchone $>$ nerolidol order. Additionally, due to the presence of ethanol, it gives noticeable release $(22.31 \mu \mathrm{g} /$ $\mathrm{cm}^{2} / \mathrm{h}^{1 / 2}$ ) from invasomes as compared with other limonene concentrations (1\% and $1.5 \%$ ). The ex vivo skin permeation study on abdominal skin (male Wistar rat) has been carried out using Franz diffusion cell. The 4\% of ethanol and $0.5 \%$ limonene based curcumin invasomes accomplished the superior permeation (8.11 times) as compared with the control. The synergetic effect of ethanol and terpene enhances the permeation of curcumin across the membrane [43]. Consequently, for solubility enhancement of curcumin, the cyclodextrin and hydroxypropyl $\beta$ cyclodextrin complex can be a suitable approach, and $0.5 \%$ limonene containing invasome can be used as a carrier for curcumin delivery through the skin. It is well known that microneedles are majorly used for the delivery of drug molecules, proteins, vaccines, etc. along with various forms. Generally, microneedles are significantly increased the skin permeability and deliver the drug across the SC. In 2009, Badran and group claimed that the invasion is a more effective technique for the delivery of a hydrophilic drug to the local/systemic applications. In this study, the drugloaded (carboxyfluorescein and radiolabelled mannitol, as a model drug) invasomes has been prepared using $1 \% \mathrm{w} / \mathrm{v}$ terpene (cineole: citral: D-limonene 45:45:10 v/v) mixture as a penetration enhancer and $13.3 \% \mathrm{w} / \mathrm{v}$ ethanolic phospholipid solution (equivalent to $10 \%$ soybean phosphatidylcholine) via mechanical dispersion. Herein, the terpene mixture vortexed $(5 \mathrm{~min})$ with ethanolic phospholipid at room temperature to obtain a clear solution. After that, the aqueous phase was added into the organic phase under constant vertexing that resulted in large multilamellar invasomes. Finally, the drug-loaded invasomes extruded using a polycarbonate membrane $(400-50 \mathrm{~nm})$ that provides the nanosized uniform invasome vesicles. Interestingly, the average size of the drug (labeled mannitol and carboxyfluorescein) loaded invasomes was found to be $123.6 \mathrm{~nm}$ and 105.5, respectively. Furthermore, the PDI value was found to be 0.08 for both drug-loaded formulations, which indicated that the high homogeneity of invasomes. The skin penetration study was performed on human abdominal skin obtained after plastic surgery that divulged the use of a dermaroller with invasome increased penetration and permeation rate of a hydrophilic drug as compared with the aqueous drug solution. Due to the presence of terpenes and ethanol increase, the interaction with the SC lipidic layers, which enhanced the drug penetration rate. The hydrophilic agent was successfully encapsulated into the core and the outer phase was water. Thus, the flexible invasome enhanced the penetration rate of active dissolved in the external water phase. Without treatment of a dermaroller, it showed less penetration of invasome-loaded mannitol across the membrane as compared with a dermaroller. The appropriate microneedle length selection gives an excellent penetration rate [32]. In conclusion, the microneedles assembly and its geometry actively influence the penetration of microneedles and consequently show an effect on the delivery of active to the selected site of application. Hence, using a dermaroller with appropriate geometry for the delivery of invasome-loaded active pharmaceutical ingredients would be a promising tool for the efficient delivery of hydrophilic/ hydrophobic molecules through the skin.

To summarize, new invasomes having perceptible pharmaceutical applications, particularly for hydrophobic and hydrophilic agents viz. antioxidant, anticancer agent, antihypertensive agent, anti-acne, anti-inflammatory, antibiotic, etc. delivery by way of the skin route. The summary of pharmaceutical applications of invasome is depicted in Table 2. 


\section{Pharmacokinetics perspective of invasomes}

From its commencement, the drug-loaded system to be explored for in vivo study that required the superior pharmacokinetic parameters, which includes active agent delivery to the specific site, circulation time in blood, proper absorption, access to the target site, half-life clearance, etc. [55]. And we witnessed that the drugs which possessed the above remarkable properties are considered for practical applications [56]. The literature revealed that it is the uniqueness of invasome which is emerging as an option designed for various pharmaceutical applications [30]. Interestingly, the upcoming need for basic information on pharmacological and toxicological aspects is a major critical step in the development of each pharmaceutical product. Therefore, the correlation of in vivo biodistribution of invasomes and its pharmacokinetics can be a major key principle intended for designing of invasomes for targeted delivery as a therapeutic application [57]. Generally, the biodistribution and pharmacokinetics of invasomes are depending on different physicochemical properties including vesicle size, shape, vesicle aggregation, solubility, penetration enhancers, and chemical compositions, etc.,[12]. It can be modified using different strategies such as terpene mixture utilization, use of microneedle strategy, iontophoresis, etc. Abundant data demonstrated that for adequate bioavailability, the invasome should have sufficient absorption from the site of application [2]. Briefly, the administration of the invasomes for delivering the active pharmaceutical ingredient has been used for both the skin and cellular membrane administration. The absorption of invasome majorly depends upon the compositions and types of penetration enhancers, lipids, different advanced strategies (including dermaroller, iontophoresis), etc. It is confirmed by using unique types of vesicles and their conventional dosage form. Additionally, invasomal formulation can be applied for both local and systemic applications. Some studies reported that the drug is accumulated in the local tissue/absorbed by systemic circulation [2,21]. Overall, the numbers of studies have also been performed to conclude the pharmacokinetics of invasomes, out of these important studies have been discussed briefly in Table 3 .

\section{Expert opinion}

The significant development in the area of invasome vesicles based drug delivery systems attracted the attention of researchers and scientists working in the field of drug delivery. Plenty of literature claimed that invasomes nanoformulations can be helped to step towards the best drug upshot while holding related toxicity to a minimum; this means successful drug treatment that has "always been sought." In short, the application of invasomes on skin surface proved to be effective in the topical delivery of a variety of drugs. Interestingly, invasomes enhances the skin permeation and reduces the side effect associated with the use of the drug as a low dose of a drug is required. Besides, invasomes have been widely used in delivering synthetic drugs (such as dapsone) as well as phytoconstituents (such as curcumin). It is worthy to mention that, invasomes can be used for site-specific and systemic drug delivery. As a topical carrier for local and systematic drug delivery, invasomes are exhibiting various applications, such as providing effective skin targeting, as it enhances the penetration of drugs across the SC and deposited in epidermal as well as dermal layers. To design the pioneered invasomes, several types of terpenes materials have been reported to be used in invasomes with different concentrations range along with consideration safety and toxicity issues. In the future, there is a prerequisite to establishing general criteria to select a common concentration range and types of terpene for the construction of invasomes. Despite the availability of numerous synthesis strategies, the scalability and reproducibility of invasomes is a major concern in nanoformulations. It may due to the nonavailability of regulatory guidance on manufacturing requirements and subsequent validation of the process to assure uniformity of invasomal quality products. As a formulation scientist, these issues related to fabrication remains unanswered. Also, improved development of invasomes can be attributed to the issue of simple and economical formulation conditions in the pharmaceutical industry being scaled up. Numerous safety trials on applications of invasomes in the delivery of drugs, on animal (preclinical) and small population groups (clinical), are needful for victorious commercialization of a range of invasome based products. While there is a lot to be done, likely, we are still far from starting with this exciting area of pharmaceuticals. There is more than fascinating biomedical promise emerging from invasomebased nanomedicines.

\section{Conclusion}

Several pharmaceutical active ingredients and chemical molecules are highly potent, but they are less active therapeutically. They can be targeted by using invasomes as a novel carrier because of its exceptional and tunable properties. Invasomes have been made their victorious entry into the pharmaceutical application research arena in 2002 with the development of cyclosporine-loaded invasomes. From the viewpoint of skin barrier properties and drawbacks, invasomes are an exceptional alternative for dermal, transdermal, and subcutaneous applications. Invasomes significantly improved the pharmacokinetic parameters since drug encapsulation. Moreover, the efficacy of the invasome dosage form depends upon the penetration rate, the ability to deliver the actives to the 
Table 3 Pharmacokinetic study of invasomes

Sr. Study Result Ref.

no.

1. Dragicevic-Curic et al. developed the temoporfin (hydrophobic drug) loaded invasomes using a ratio of limonene, citral, and cineole as a penetration enhancer and tested the in vitro permeation of temoporfin into abdominal human skin using Franz diffusion cells.

2. Yet another study showed the temoporfin (hydrophobic drug) invasomes efficacy for topical application onto the skin of mice bearing the subcutaneously implanted human colorectal tumor HT29.

3. In this study, isotretinoin-loaded invasomal gel was developed to achieve targeted delivery of drugs to the pilosebaceous follicular unit.

4. Chen et al. accomplished ferulic acid-loaded invasomes for efficient delivery to the targeted skin part and compared with other vesicular systems.

5. In 2013, Prasanthi et al. developed the finasteride-loaded invasomal vesicle for transdermal delivery using the iontophoretic technique.

6. Kamran et al. reported the olmesartan-loaded novel nano-invasome using b-citronellene as a permeation enhancer to increases the bioavailability and biological half-life of a drug.

7. In 2014, Lakshmi et al. developed the curcumin-loaded cyclodextrin and hydroxypropyl $\beta$-cyclodextrin complex and incorporated it into invasome and then formulate the hydroxypropyl cellulose gel as a transdermal formulation and observed for ex vivo skin permeation studies using rat abdominal skin.

8. In 2018, El-Nabarawi et al. investigated the dapsone-loaded invasome using unique types of terpene through thin-film hydration and tested for the ability to in vivo delivery of the dapsone through the skin using Wistar rats.

9. In this study, the nanosized avanafil-loaded invasomes is used to increase the transdermal delivery of drug and consequently bioavailability of avanafil.

10. Tolterodine tartrate-loaded invasomes were prepared using different terpene, soya lecithin, ethanol, and tested for transdermal penetrations.

11. In 2017, Qadri et al. developed the isradipine-loaded invasome using Phospholipon ${ }^{\oplus}$ 90G, b-citronellene (terpene), and ethanol by conventional thin-layer evaporation technique and tested transdermal flux, bioavailability, etc.

12. Ultra-flexible invasomes for transdermal delivery of capsaicin loaded has been designed using Comperlan ${ }^{\circledR} \mathrm{KD}$ and D-limonene as a potential penetration enhancer.

13. The phenylethyl resorcinol-loaded invasomes have been designed by Amnuaikit et al. for topical delivery of phenyl ethyl resorcinol.

14. Anastrozole was loaded into invasomes and formulate the invasomal gel using sodium carboxymethyl cellulose for effective transdermal delivery of the anticancer drug in the treatment of breast cancer.

15. The hydrophilic model drug-loaded invasomal formulations have been designed and tested for skin permeation and penetration using microneedles based device (Dermaroller ${ }^{\circledR}$ ) with different needle lengths.
Invasomes containing 1\% (w/v) cineole showed the highest skin penetration enhancement of temoporfin. Also, it gives exorbitant amounts of temoporfin the SC and deeper skin layers.

The results showed a benefit above the previously reported delivery of temoporfin systems for delivering anticancer drugs more efficiently to the subcutaneous layer of skin.

The developed gel formulation exhibited a biphasic ex vivo permeation behavior (fast permeation and zero-order) and after $2 \mathrm{~h}$ the permeation slowed down without altering the order of permeation. Also, it showed the penetration of invasomes to the deep dermal region of the skin for pilosebaceous targeting.

Tween 80-based invasomes showed improved permeation profiles and enhanced skin deposition of ferulic acid.

The optimized invasomal formulation enhanced permeation rate, flux, bioavailability, and half-life using the iontophoretic technique.

The confocal laser microscopy of rat skin revealed the drug was eventually distributed and permeated deep into the skin. It showed 1.15 times improvement in bioavailability concerning the control olmesartan formulation in Wistar rats.

Invasomal preparation exhibited an enhanced permeation rate (8.11 times) as compared with the control. In vivo diffusion studies showed the high cumulative drug permeation rate, steady-state transdermal flux as compared with the control formulation.

The in vivo skin deposition amount of dapsone was increased (4.11 $\mathrm{mcg} / \mathrm{cm} 2)$ as compared with alcoholic drug solution $(1.71 \mu \mathrm{g} / \mathrm{cm} 2)$. Interestingly AUC of dapsone-loaded invasomes was nearly 2 -fold greater than dapsone solution

The results of the invasomal film showed enhanced ex vivo permeation with an enhancement factor of 2.514. Also, a 4-fold increase in relative bioavailability compared with the raw avanafil film.

The iontophoretic drug transport showed that the permeability of tolterodine tartrate released from invasomes was higher compared with that of free drug proving the additive effect of invasomes and iontophoresis. Also, invasome formulation containing limonene showed high penetration because of its lipophilicity and low boiling point.

It showed an enhanced permeation of isradipine invasomes to the deeper layers of the rat skin. It was found more effective, and it reduces the 20\% blood pressure by improved permeation through Wistar rat skin.

The skin permeation study showed that invasomes exhibited significantly higher permeation as compared with the conventional liposomes and $0.15 \%$ capsaicin in ethanolic solution (commercial product).

It showed efficiency to deliver phenylethyl resorcinol into the deep skin in both quantity and effectiveness.

Ex vivo permeation study showed an enhancement of transderma flux and the skin deposition of the drug (73\%) have been studied on male Wistar rat skin. Also, prepared invasomal gel showed firstorder kinetics behavior with Higuchi model-dependent kinetics.

Interestingly, it has been observed the skin perforation with the Dermarollers ${ }^{\circledast}$ enhanced drug (carboxyfluorescein and mannitol) penetration and permeation for both developed invasomal formulations as compared with aqueous drug solutions. 
Table 3 Pharmacokinetic study of invasomes (Continued)

\begin{tabular}{|c|c|c|c|}
\hline $\begin{array}{l}\text { Sr. } \\
\text { no. }\end{array}$ & Study & Result & Ref. \\
\hline 16. & $\begin{array}{l}\text { Verma et al. developed the lipophilic drug (cyclosporine A) loaded } \\
\text { invasomes to enhance the penetration of cyclosporine A. }\end{array}$ & $\begin{array}{l}\text { Interestingly, it revealed an in vitro study on human skin, the } \\
\text { invasomes provided a significantly higher amount of cyclosporine A } \\
\text { in the deeper layers of human skin (feasible epidermis and dermis). }\end{array}$ & {$[12]$} \\
\hline 17. & $\begin{array}{l}\text { Shah et al. developed and compared the ability of drug-loaded ves- } \\
\text { icular systems (cationic LeciPlex, invasomes, and conventional lipo- } \\
\text { some) to deliver the drugs deep into the skin. }\end{array}$ & $\begin{array}{l}\text { The ex vivo study showed the invasomes increased the delivery of } \\
\text { azelaic acid at the site of inflammation of acne as compared with } \\
\text { other vesicular systems, which resulted in the fast therapeutic } \\
\text { action. }\end{array}$ & [25] \\
\hline 18. & $\begin{array}{l}\text { In 2009, Mura and co-authors developed the minoxidil-loaded inva- } \\
\text { somes using soya lecithin, and different penetration enhancer to } \\
\text { achieve efficient (trans) dermal delivery. }\end{array}$ & $\begin{array}{l}\text { In vitro diffusion through newborn pig skin showed the developed } \\
\text { invasomes achieved the significant improvement of minoxidil } \\
\text { deposition in the skin and consequently improving cutaneous drug } \\
\text { bioavailability. }\end{array}$ & [35] \\
\hline
\end{tabular}

targeted site and low toxicity, etc. Various factors like lipid to drug ratio, drug concentration, terpene ratio and its nature, vesicle size, entrapment efficiency, etc. consideration is a must for invasome formulation development. Additionally, invasomes can be a suitable carrier for the delivery of hydrophobic and hydrophilic drugs. Thus, an invasome-based drug delivery approach can fruitfully overcome the drawbacks of formerly emerged dosage forms and techniques. In the future, it would be a leading nano-formulation approach and surely it will take a position in novel delivery systems.

\section{Abbreviations}

SC: Stratum corneum; PBS: Phosphate buffer saline; SEM: Scanning electron microscopy; EM: Transmission electron microscopy; SUVs: Small unilamellar vesicles; LUVs: Large unilamellar vesicle; DLS: Dynamic light scattering; PDI: Polydispersity index; CsA, CyA: Cyclosporine A

\section{Acknowledgements}

The authors are thankful to the institute for providing facilities such as a library, journals, and internet sources for writing the article.

\section{Authors' contributions}

SN: conceptualization, methodology, writing—original draft, writing-review and editing, visualization; SD: writing —original draft, writing-review and editing. All authors gave their individual critical revision and final approval of the version to be submitted.

\section{Funding}

Not applicable.

\section{Availability of data and materials}

All data and materials are available on request.

\section{Ethics approval and consent to participate}

Not applicable.

\section{Consent for publication}

Not applicable

\section{Competing interests}

The authors declare that they have no conflict of interest.

\section{Author details}

${ }^{1}$ Department of Pharmaceutical Chemistry, H. R. Patel Institute of Pharmaceutical Education and Research, Shirpur, Dhule, MS 425405, India.

${ }^{2}$ Institute of Chemical Technology, Matunga, Mumbai, MS 400019, India.
Received: 8 September 2020 Accepted: 18 November 2020

Published online: 13 December 2020

\section{References}

1. Dragicevic N, Maibach H (2018) Combined use of nanocarriers and physical methods for percutaneous penetration enhancement. Adv Drug Deliv Rev 127:58-84

2. Lakshmi P, Kalpana B, Prasanthi D (2013) Invasomes-novel vesicular carriers for enhanced skin permeation. Sys Rev Pharm 4:26

3. Brown MB, Martin GP, Jones SA, Akomeah FK (2006) Dermal and transdermal drug delivery systems: current and future prospects. Drug Deliv 13:175-187

4. Bouwstra J, Honeywell-Nguyen P (2002) Skin structure and mode of action of vesicles. Adv Drug Deliv Rev 54:S41-S55

5. Elias PM (1983) Epidermal lipids, barrier function, and desquamation. J Invest Dermatol 80(1):44 s-49s

6. Bouwstra J, Dubbelaar F, Gooris G, Ponec M (2000) The lipid organisation in the skin barrier. Acta Derma Venereol 208:23-30

7. Cross S, Roberts M (2004) Physical enhancement of transdermal drug application: is delivery technology keeping up with pharmaceutical development? Curr Drug Deliv 1:81-92

8. Bouwstra JA, Honeywell-Nguyen PL, Gooris GS, Ponec M (2003) Structure of the skin barrier and its modulation by vesicular formulations. Prog Lipid Res 42:1-36

9. Zhou X, Hao Y, Yuan L, Pradhan S, Shrestha K, Pradhan O, Liu H, Li W (2018) Nano-formulations for transdermal drug delivery: a review. Chin Chem Lett 29:1713-1724

10. Chauhan P, Tyagi BK (2018) Herbal novel drug delivery systems and transfersomes. J Drug Deliv Ther 8:162-168

11. Karimi N, Ghanbarzadeh B, Hamishehkar H, Keyvani F, Pezeshki A, Gholian MM (2015) Phytosome and liposome: The beneficial encapsulation systems in drug delivery and food application. Appl Food Biotechnol 2(3):17-27

12. Dragicevic N, Verma DD, Fahr A (2016). Invasomes: Vesicles for enhanced skin delivery of drugs. In: Dragicevic N., Maibach H. (eds) Percutaneous Penetration Enhancers Chemical Methods in Penetration Enhancement. Springer, Berlin, Heidelberg, pp 77-92. https://doi.org/10.1007/978-3-662-47862-2_5.

13. Prasanthi D, Lakshmi $P$ (2012) Vesicles-mechanism of transdermal permeation: a review. Asian J Pharm Clin Res 5:18-25

14. Akhtar N (2014) Vesicles: a recently developed novel carrier for enhanced topical drug delivery. Curr. Drug Deliv 11:87-97

15. Dragicevic-Curic N, Scheglmann D, Albrecht V, Fahr A (2009) Development of different temoporfin-loaded invasomes-novel nanocarriers of temoporfin: Characterization, stability and in vitro skin penetration studies. Colloids Surf B: Biointerfaces 70:198-206

16. Ashtikar M, Nagarsekar K, Fahr A (2016) Transdermal delivery from liposomal formulations-Evolution of the technology over the last three decades. J Control Release 242:126-140

17. Katare OP, Raza K, Singh B, Dogra S (2010) Novel drug delivery systems in topical treatment of psoriasis: rigors and vigors. Indian J Dermatol Venereol Leprol 76(6):612-621

18. Nava-Arzaluz MG, Piñón-Segundo E, Ganem-Rondero A (2019). Lipid nanocarriers as skin drug delivery systems. Nanopart Pharmacother: William 
Andrew Publishing, pp 311-390 Elsevier. https://doi.org/10.1016/B978-0-12816504-1.00007-7.

19. Milewski M, Brogden NK, Stinchcomb AL (2010) Current aspects of formulation efforts and pore lifetime related to microneedle treatment of skin. Expert Opin Drug Deliv 7:617-629

20. Moradi L, Javanmardi S, Abolmaali S, Mohammadi Samani S (2019) Passive enhancement of transdermal drug delivery: Lipid-based colloidal carriers as an emerging pharmaceutical technology platform. Trends Pharmacol Sci 5: 25-40

21. Ashtikar M, Langelüddecke L, Fahr A, Deckert V (2017) Tip-enhanced Raman scattering for tracking of invasomes in the stratum corneum. Biochimica et Biophysica Acta (BBA)-General Subjects 1861(11):2630-2639

22. Romero EL, Morilla MJ (2015) Ultradeformable phospholipid vesicles as a drug delivery system: a review. Res Reports Transderm Drug Deliv 2015(4): 55-69

23. Bommannan D, Potts RO, Guy RH (1991) Examination of the effect of ethanol on human stratum corneum in vivo using infrared spectroscopy. J Control Release 16:299-304

24. Dragicevic-Curic N, Friedrich M, Petersen S, Scheglmann D, Douroumis D, Plass W, Fahr A (2011) Assessment of fluidity of different invasomes by electron spin resonance and differential scanning calorimetry. Int J Pharm 412:85-94

25. Shah SM, Ashtikar M, Jain AS, Makhija DT, Nikam Y, Gude R, Steiniger F, Jagtap AA, Nagarsenker MS, Fahr A (2015) LeciPlex, invasomes, and liposomes: A skin penetration study. Int J Pharm 490(1-2):391-403

26. Qadri GR, Ahad A, Aqil M, Imam SS, Ali A (2017) Invasomes of isradipine for enhanced transdermal delivery against hypertension: formulation, characterization, and in vivo pharmacodynamic study. Artif Cell Nanomed B 45:139-145

27. Dwivedi M, Sharma V, Pathak K (2017) Pilosebaceous targeting by isotretenoin-loaded invasomal gel for the treatment of eosinophilic pustular folliculitis: optimization, efficacy and cellular analysis. Drug Dev Ind Pharm 43:293-304

28. Dragicevic-Curic N, Scheglmann D, Albrecht V, Fahr A (2008) Temoporfinloaded invasomes: development, characterization and in vitro skin penetration studies. J Control Release 127:59-69

29. Neubert RH (2011) Potentials of new nanocarriers for dermal and transdermal drug delivery. Eur J Pharm Biopharm 77:1-2

30. Prasanthi D, K Lakshmi P (2013) lontophoretic transdermal delivery of finasteride in vesicular invasomal carriers. Pharm Nanotechnol 1:136-150

31. Akiladevi D, Basak S (2010) Ethosomes-a noninvasive approach for transdermal drug delivery. Int J Curr Pharm Res 2:1-4

32. Badran M, Kuntsche J, Fahr A (2009) Skin penetration enhancement by a microneedle device (Dermaroller ${ }^{\circledR}$ ) in vitro: Dependency on needle size and applied formulation. Eur J Pharm Sci 36:511-523

33. Dragicevic-Curic N, Gräfe S, Albrecht V, Fahr A (2008) Topical application of temoporfin-loaded invasomes for photodynamic therapy of subcutaneously implanted tumours in mice: a pilot study. J Photochem Photobiol B 91:4150

34. Vidya K, Lakshmi P (2019) Cytotoxic effect of transdermal invasomal anastrozole gel on MCF-7 breast cancer cell line. J Appl Pharm Sci 9:050058

35. Mura S, Manconi M, Sinico C, Valenti D, Fadda AM (2009) Penetration enhancer-containing vesicles (PEVs) as carriers for cutaneous delivery of minoxidil. Int J Pharm 380:72-79

36. El-Nabarawi MA, Shamma RN, Farouk F, Nasralla SM (2018) Dapsone-loaded invasomes as a potential treatment of acne: Preparation, characterization, and in vivo skin deposition assay. AAPS PharmSciTech 19:2174-2184

37. Duangjit S, Nimcharoenwan T, Chomya N, Locharoenrat N, Ngawhirunpat T (2016) Design and development of optimal invasomes for transdermal drug delivery using computer program. Asian J Pharm Sci 11:52-53

38. Amnuaikit T, Limsuwan T, Khongkow P, Boonme P (2018) Vesicular carriers containing phenylethyl resorcinol for topical delivery system; liposomes, transfersomes and invasomes. Asian J Pharm Sci 13:472-484

39. Ahmed OA, Badr-Eldin SM (2019) Development of an optimized avanafilloaded invasomal transdermal film: Ex vivo skin permeation and in vivo evaluation. Int J Pharm 570:118657

40. Kamran M, Ahad A, Aqil M, Imam SS, Sultana Y, Ali A (2016) Design, formulation and optimization of novel soft nano-carriers for transdermal olmesartan medoxomil delivery: in vitro characterization and in vivo pharmacokinetic assessment. Int J Pharm 505:147-158
41. Kalpana B, Lakshmi P (2013) Transdermal permeation enhancement of Tolterodine Tartrate through invasomes and iontophoresis. Der Pharm Lett 5:119-126

42. Chen M, Liu X, Fahr A (2010) Skin delivery of ferulic acid from different vesicular systems. J Biomed Nanotechnol 6:577-585

43. Lakshmi P, Mounica V, Manoj KY (2014) Prasanthi D (2014) Preparation and evaluation of curcumin invasomes. Int J Drug Deliv 6:113-120

44. Dragicevic-Curic N, Gräfe S, Gitter B, Fahr A (2010) Efficacy of temoporfinloaded invasomes in the photodynamic therapy in human epidermoid and colorectal tumour cell lines. J Photochem Photobiol B 101:238-250

45. Verma P, Pathak K (2012) Nanosized ethanolic vesicles loaded with econazole nitrate for the treatment of deep fungal infections through topical gel formulation. Nanomedicine 8:489-496

46. Chung G, Oh SB (2013) Eugenol as local anesthetic. Natural Products: Phytochemistry, Botany and Metabolism of Alkaloids, Phenolics and Terpenes, pp 4001-4015

47. Vaddi H, Ho P, Chan Y, Chan S (2002) Terpenes in ethanol: haloperido permeation and partition through human skin and stratum corneum changes. J Control Release 81:121-133

48. Sinico C, Fadda AM (2009) Vesicular carriers for dermal drug delivery. Expert Opin Drug Deliv 6:813-825

49. Verma D, Verma S, McElwee K, Freyschmidt-Paul P, Hoffman R, Fahr A (2004) Treatment of alopecia areata in the DEBR model using Cyclosporin A lipid vesicles. Eur J Dermatol 14:332-338

50. Al-Lawati H, Aliabadi HM, Makhmalzadeh BS, Lavasanifar A (2018) Nanomedicine for immunosuppressive therapy: achievements in pre-clinical and clinical research. Expert Opin Drug Deliv 15:397-418

51. Nangare S, Jadhav N, Ghagare P, Muthane T (2020) Pharmaceutical applications of electrospinning. Ann Pharm Fr 78(1):1-11

52. Hosking A-M, Juhasz M, Mesinkovska NA (2019) Complementary and alternative treatments for alopecia: a comprehensive review. Skin appendage disorders. Skin Appendage Disord 5:72-89

53. Nangare S, Dhananjay B, Mali R, Shitole M (2020) Development of novel freeze-dried mulberry leaves extract-based transfersomal gel. Turk J Pharm Sci. https://doi.org/10.4274/tjps.98624

54. Shitole M, Dugam S, Tade R, Desai N, Nangare S (2020) Progress in erectile dysfunction therapy through drug delivery system. Thai J Pharm Sci 44:7281

55. Al Faraj A, Shaik AS, Al Sayed B (2015) Preferential magnetic targeting of carbon nanotubes to cancer sites: noninvasive tracking using MRI in a murine breast cancer model. Nanomedicine 10:931-948

56. Liang F, Chen B (2010) A review on biomedical applications of single-walled carbon nanotubes. Curr Med Chem 17:10-24

57. Mbah CC, Builders PF, Attama AA (2014) Nanovesicular carriers as alternative drug delivery systems: ethosomes in focus. Expert Opin Drug Deliv 11:45-59

\section{Publisher's Note}

Springer Nature remains neutral with regard to jurisdictional claims in published maps and institutional affiliations.

\section{Submit your manuscript to a SpringerOpen ${ }^{\circ}$ journal and benefit from:}

- Convenient online submission

- Rigorous peer review

- Open access: articles freely available online

- High visibility within the field

- Retaining the copyright to your article

Submit your next manuscript at $\boldsymbol{\nabla}$ springeropen.com 